\begin{tabular}{|l|l|c|l|}
\hline Eiszeitalter $u$. Gegenwart & $\mathbf{4 0}$ & $\begin{array}{c}1-19 \\
17 \mathrm{Abb} ., 2 \mathrm{Tab} .\end{array}$ & Hannover 1990 \\
\hline
\end{tabular}

\title{
Jungpleistozäne und holozäne Skelettfunde von Bos primigenius und Bison priscus aus Deutschland und ihre Bedeutung für die Zuordnung isolierter Langknochen
}

\author{
THOMAS MARTIN *)
}

\author{
Upper Pleistocene, Holocene, Ruminantia, Bos primigenius, Bison priscus, \\ skeletons, skulls, extremities, humerus, radius, femur, tibia, biometry, taxonomy \\ Lower Saxony, Northern German Hills, Hesse, \\ Upper Rhine Valley, North Rhine-Westphalia, Rhine Westphalian Basin, \\ TK $3728,4905,6116,6216$
}

\begin{abstract}
Kurzfassung: Drei Auerochsenskelette (Bos primigenius) und ein Bisonskelett (Bison priscus) aus dem Jungpleistozän bzw. Holozän Deutschlands werden osteologisch beschrieben. Gattungsunterschiede an den Langknochen werden an den mit Hilfe des Schädels sicher bestimmten Skelettfunden auf ihre Zuverlässigkeit hin überprüft. In die Untersuchung wird Vergleichsmaterial von Bos primigenius und Bison priscus aus dem Pleistozän von England sowie von Bison div. sp. aus Nordamerika einbezogen.
\end{abstract}

Schlüsselmerkmale für eine Unterscheidung von Bos primigenius und Bison priscus existieren nicht. Die in einer früheren Arbeit an Material aus den Schottern der nördlichen Oberrheinebene (MARTIN 1987) ausgearbeiteten Merkmalskombinationen konnten als brauchbar bestätigt werden, wobei sich erneut zeigte, daß sehr zierliche Knochen in ihren Merkmalsausprägungen abweichen können. Die Unterscheidungsmerkmale lassen sich generell auch auf den nordamerikanischen Bison antiquus und Bison bison erfolgreich anwenden, sofern die Knochen in derselben Größenklasse wie die von Bison priscus liegen.

\section{[Skeleton Finds of Bos primigenius and Bison priscus from the Late Pleistocene and Holocene of Germany and their Importance for the Determination of Isolated Long Bones]}

Abstract: Three skeletons of Bos primigenius and one of Bison priscus from the late Pleistocene and Holocene of Germany are described osteologically. The utility of distinctive character combinations at the long bones of the two genera is proven at by their skulls undoubtedly determined skeletons. For comparison, long bones of Bos primigenius and Bison

*) Anschrift des Verfassers: Dipl.-Geol. TH. MARTIN, Institut für Paläontologie der Rheinischen Friedrich-WilhelmsUniversität, Nußallee 8, D - 5300 Bonn 1. priscus from England, and of Bison div. sp. from North America are included in the investigation. Key characters for a distinction of Bos primigenius and Bison priscus do not exist. The combinations of distinctive characters worked out earlier (MARTIN 1987) are confirmed as usable for a distinction of these bovids. Generally they can also be used for the North American Bison bison and Bison antiquus, if the bones are in the same size range as these of Bison priscus.

\section{Inhalt}

1. Einleitung

2. Methodisches, Abkürzungen und Terminologie

3. Die Skelettfunde

3.1. Der Düsseldorfer Auerochse

3.2. Der Braunschweiger Auerochse

3.3. Auerochsenteilskelett von Erfelden

3.4. Bisonskelett aus Groß-Rohrheim

3.5. Vergleichsmaterial aus Großbritannien

3.6. Vergleichsmaterial aus Nordamerika

4. Merkmalsausprägung an den Extremitätenknochen
4.1. Humerus
4.2. Radius
4.3. Femur
4.4. Tibia

5. Vergleich mit Bison div. sp. aus dem nordamerikanischen Jungpleistozän

6. Zusammenfassung

7. Danksagung

8. Schriftenverzeichnis 


\section{Einleitung}

Verglichen mit der Vielzahl von Einzelknochen sind bisher nur wenige zusammengehörige Skeletteile von Boviden aus dem europäischen Jungpleistozän bekannt geworden. Die exakte Zuweisung isolierter Skelettelemente zu Bos oder Bison bereitet Schwierigkeiten, da sich beide Gattungen am postkranialen Skelett nur durch feine, zumeist graduelle Unterschiede voneinander abtrennen lassen (BOJANUS 1828, RÜTIMEYER 1862, SCHERTZ 1936, LEHMANN 1949, BIBIKOVA 1958, STAMPFL 1963). In einer früheren Arbeit (MARTIN 1987) konnte gezeigt werden, daß die Unterscheidung von Bos primigenius und Bison priscus an isolierten Langknochen nur mit mehreren Merkmalen in Kombination getroffen werden kann; die früher betriebene Suche nach Schlüsselmerkmalen erwies sich als erfolglos. Mit Hilfe von Merkmalskombinationen lassen sich bei guter Erhaltung ca. $80 \%$ der isolierten Langknochen bestimmen. Daher sind Skelettfunde, bei denen der Schädel erhalten ist, besonders wertvoll: Denn der Schädel ist das einzige Skelettelement, an dem sich Bos primigenius und Bison priscus auf dem ersten Blick sicher unterscheiden lassen (Abb. 1). Bei Bos primigenius überragen die Basen der Hornzapfen, die durch einen leicht eingezogenen Grat verbunden sind, das Hinterhaupt; bei Bison priscus dagegen erhebt sich das Hinterhaupt weit über die wesentlich tiefer und weiter vorn am Schädel sitzenden Hornbasen. Bison priscus zeichnet sich durch seitwärts und nach oben gebogene Hörner aus, wogegen sie beim Auerochsen eine charakteristische dreifache Biegung zeigen, die erst seitwärts, dann nach oben und schließlich nach vorne verläuft. Allein Skelettfunde mit Schädel ermöglichen es, die - not- gedrungen oft an isolierten Knochen statistisch ermittelten - Bestimmungsmerkmale zu „eichen”.

In dieser Arbeit sollen Skelettfunde der beiden Boviden-Gattungen vorgestellt und diskutiert werden; dies jedoch nicht, um lediglich deren Existenz anzuzeigen, sondern um die bisher bekannten Unterscheidungsmerkmale weiter abzusichern und in ihrer Brauchbarkeit zu überprüfen. Darüber hinaus wird untersucht, ob die Bison priscus kennzeichnenden Merkmalsausprägungen auch beim nordamerikanischen Bison beobachtet werden können. Auf die Auerochsen-Funde aus Dänemark (DEGERBøL \& FRED. SKILD 1970, AARIS-SøRENSEN \& BRINCH PETERSEN 1986) kann hier nicht eingegangen werden.

Die Gattung Bison tritt in Mitteleuropa erstmals vor ca. 1 Mio Jahren auf, z. B. in den Hochterrassen-Sanden der Werra bei Meiningen (Südthüringen), in denen der Beginn des Jaramillo-Events nachgewiesen wurde (WIEGANK 1983, KAHLKE 1982). Bison priscus ist in den Ablagerungen von Voigtstedt in Thüringen (FISCHER 1965) und Süßenborn bei Weimar (FLEROV 1969), die dem klassischen Cromer Forest Bed gleichgesetzt werden können (STUART 1982, KOENIGSWALD \& TOBIEN 1987), gut belegt. In den jüngeren Interglazialen des Cromer-Komplexes finden sich zwei Bison-Formen, der kurzhornige Waldbison (Bison schoetensacki) und der langhornige Steppenbison (Bison priscus), der in Mitteleuropa bis zum Ende des Würm vorkommt (KURTÉN 1968). Da Bison schoetensacki im mittleren Pleistozän verschwindet, kommt er nicht als direkter Vorfahr von Bison bonasus, des rezenten Waldwisent, in Frage (KURTÉN 1968). Bison bonasus wird heute mit dem nordamerikanischen

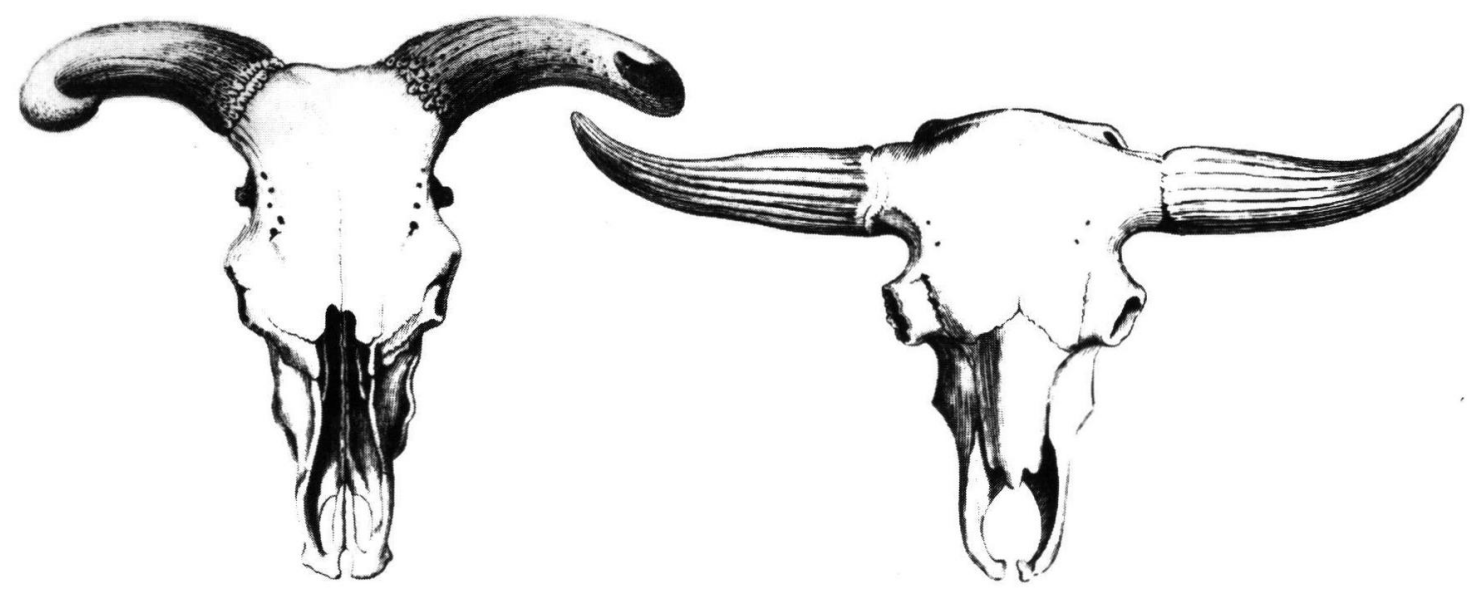

Abb. 1: Am Schädel lassen sich Bos primigenius (links) und Bison priscus (rechts) durch die unterschiedliche Form des Hinterhauptes und der Hornzapfen gut auseinanderhalten; nach CUVIER (1836). 
Bison bison von einem gemeinsamen Ursprung abgeleitet (SKINNER \& KAISEN 1947: 215).

Bison priscus ist als ein beherrschendes Faunenelement des europäischen Jungpleistozäns meist häufiger als der Auerochse (Bos primigenius), der in Mitteleuropa erstmals im Interglazial der unteren Schotter von Steinheim an der Murr (LeHMANN 1949), die dem Holstein-Interglazial gleichgestellt werden (ADAM 1954), auftaucht. Während des Mittel- und Jungpleistozäns bleibt Bos verhältnismäßig selten, erst in holozänen Ablagerungen häufen sich die Überreste (ZEUNER 1967: 176). So stammen mehrere Funde aus holozänen Torfmooren (z. B. RUUTIMEYER 1862, FIEDLER 1907, HÜNERMANN 1968).

Der Auerochse hielt sich in Mitteleuropa bis in geschichtliche Zeit und war im Mittelalter ein begehrtes Jagdwild; die letzte Kuh starb 1627 in einem Wildgatter im Wald von Jaktorow bei Warschau (KURTÉN 1968). Bos primigenius ist die Stammform unseres Hausrindes, Bos primigenius f. taurus (ZEUNER 1967: 175).

Eine Verwechslungsgefahr postkranialer Skelettelemente von Bos primigenius und Bison priscus besteht also nur bei pleistozänem Material. Die zierlichen Knochen des leicht gebauten Bison bonasus, der in Europa erst im Postglazial auftritt (KURTÉN 1968) und noch heute in einem Wildreservat im Urwald von Bialowieza (Ostpolen) lebt (MOHR 1952), sind meist problemlos von denen des wesentlich stärkeren Auerochsen zu unterscheiden.

\section{Methodisches, Abkürzungen und Terminologie}

Die Standardmaße wurden nach vON DEN DRIESCH (1976) genommen; die Meßstrecken an Schädel und Extremitätenknochen können den Abb. 2 bis 7 entnommen werden. Die Meßwerte in den Tabellen sind auf halbe mm gerundet. Die Lage der diskutierten Bestimmungsmerkmale zeigen die Abb. 8 bis 11 . Anatomische Benennungen wurden nach NICKEL et al. (1984) vorgenommen.

Die Institute und Museen, aus deren Sammlungen Material bearbeitet wurde, werden im Text unter folgenden Abkürzungen geführt:

BMNH British Museum (Natural History, Dept. of Vertebrate Paleontology), London

HLMD Hessisches Landesmuseum (GeologischPaläontologische und Mineralogische $\mathrm{Ab}$ teilung), Darmstadt; RS ist die Abkürzung für die Fossilien der Rheinschotter-Sammlung
KUMVP University of Kansas (Museum of Natural History, Dept. of Vertebrate Paleontology), Lawrence

LACM Los Angeles County Museum (hier: George C. Page Museum)

LÖMD Löbbecke-Museum and Aquarium, Düsseldorf

SNMB Staatliches Naturhistorisches Museum, Braunschweig

\section{Material}

Für die Untersuchung standen drei mehr oder weniger vollständige Skelette von Bos primigenius und eines von Bison priscus zur Verfügung.

Eines der Auerochsenskelette ist holozänen Alters und stammt aus einem Torfmoor bei Braunschweig, ein weiteres wurde in den Deckschichten der rheinischen Braunkohle gefunden. Die dunkle Färbung der Knochen weist auch hier auf einen Moorfund hin, was wahrscheinlich ebenfalls ein holozänes Alter bedeutet.

Die anderen Skelette wurden in den letzten Jahren aus den jungpleistozänen Kiesen und Sanden der nördlichen Oberrheinebene geborgen.

\subsection{Der Düsseldorfer Auerochse}

Die Angaben zu Fundort und Fundzeitpunkt des bis auf die Hinterextremitäten weitgehend vollständigen Skelettes (Abb. 12) sind unklar. Nach BosCHEINEN (1985) und MEINECKE (1985) soll es in den vierziger Jahren (dieses Jahrhunderts) im rheinischen Braunkohlenrevier bei Frimmersdorf, heute Ortsteil von Grevenbroich, $25 \mathrm{~km}$ nw' Köln, gefunden worden sein. Herr BOSCHEINEN äußerte mir gegenüber jedoch Zweifel an diesen Funddaten und wies mich darauf hin, daß GÖRGES (1912) in einem Artikel über das Löbbecke-Museum ein Skelett von Bos primigenius, gefunden bei Grevenbroich, erwähnt, das „im Laufe der letzten Jahre" zu den Sammlungen Löbbeckes, die den Grundstock des Museums bilden, hinzugekommen sei. Demnach ist das auch bei LANGER (1974) erwähnte und immer noch unbearbeitete Skelett mit großer Wahrscheinlichkeit bereits in den 40er Jahren des letzten Jahrhunderts im Raum FrimmersdorfGrevenbroich gefunden worden. Als Fundhorizonte kommen nur die pleistozänen oder holozänen Deckschichten der Braunkohle in Frage.

Das mehrfach restaurierte Skelett wird seit seiner Auffindung im Löbbecke-Museum, heute Löbbecke 

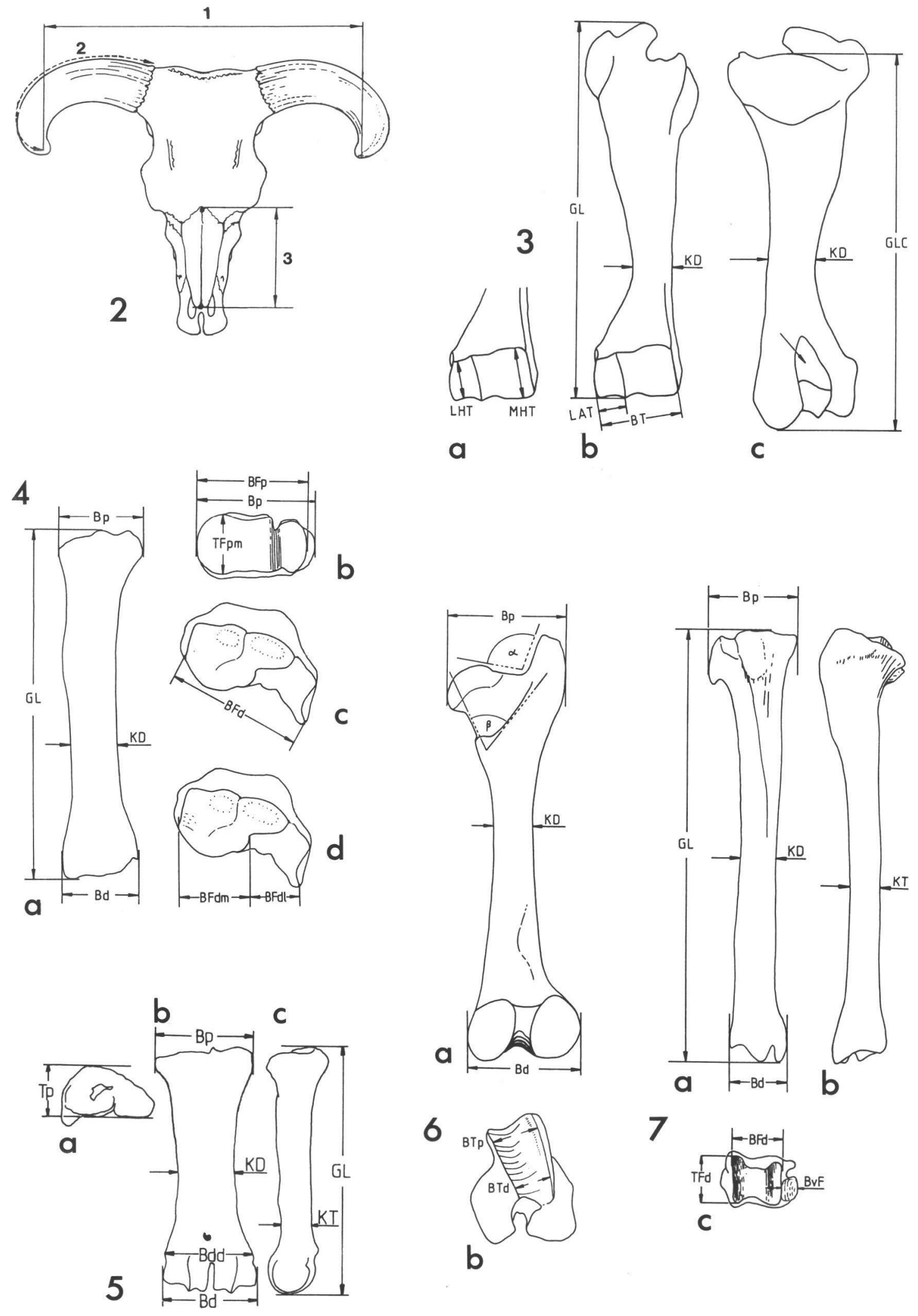
Museum und Aquarium, in Düsseldorf aufbewahrt. Leider konnte es nur in montiertem Zustand untersucht werden, was die Beurteilung einiger wichtiger Bestimmungsmerkmale verhinderte.

\section{Umfang des Skelettes}

Der Schädel ist mit beiden Hornzapfen erhalten; die Schädelmaße sind etwas geringer als die des außergewöhnlich starken Braunschweiger Auerochsen (siehe Tab. 1).

Der linke Unterkiefer (weitgehend aus Gips ergänzt) enthält $\mathrm{P}_{2}$ und $\mathrm{P}_{3}$, sowie $\mathrm{M}_{1}-\mathrm{M}_{3}$; der rechte Unterkiefer ist vollständig nachgebildet.

Vom Achsenskelett liegen 7 Halswirbel, 12 Brustwirbel mit 12 Paar Rippen, 7 Lendenwirbel und 13 Schwanzwirbel vor. Vom Schultergürtel sind beide Schulterblätter (dorsal ergänzt) vorhanden, dazu linker und rechter Humus, Radii mit Ulnae, Carpalia und Metacarpalia; die Phalangen sind ergänzt. Das Becken ist weitgehend aus Gips rekonstruiert; lediglich das Pubis und Knochenteile rund um das Acetabulum sind original. Ebenso ist das Kreuzbein zwar im Kern original, außen jedoch stark mit Gips und Kunststoff überschmiert. Die Hinterextremitäten sind durch Abgüsse von dem Auerochsenskelett des Staatlichen Naturhistorischen Museums in Braunschweig ergänzt.

\section{Alter und Geschlecht}

Der wuchtige Schädel mit den mächtigen Hornzapfen, die geschlossenen Epiphysenfugen sowie die mäßig abgekauten Zähne lassen auf einen Bullen mittleren Alters schließen. Leider ist das Becken zu schlecht erhalten, um an ihm Hinweise auf das Geschlecht ablesen zu können.

Abb. 2-7: Meßstrecken an Schädel, Metapodien und Langknochen.

Wenn nicht anders angegeben, wurden die Maße im Meßkasten genommen; $(\mathrm{S})=$ Schieblehre, $(\mathrm{Ma})=\mathrm{Maßband}$.

Abb. 2: Meßstrecken am Schädel (nach vON DEN DRIESCH 1976, verändert);

1 = Gerade Entfernung der Hornzapfenspitzen (Ma); 2 = Außenlänge eines Hornzapfens (Ma); 3 = Länge der Nasalia (S) . Durchmesser und Umfang der Hornzapfen wurden an der Perlbildung der Basis genommen.

Abb. 3: Humerus; 3a. Distale Gelenkrolle von kranial, LHT = laterale Höhe der Trochlea (S), $\mathrm{MHT}=$ mediale Höhe der Trochlea (S); 3 b. Kranialansicht, BT = Breite der Trochlea (S), GL = Größte Länge,

$\mathrm{KD}=$ Kleinster Durchmesser der Diaphyse (S), LAT = Breite des lateralen Abschnittes der Trochlea (gemessen vom Außenrand des lateralen Wulstes bis zum höchsten Punkt des mittleren Führungsgrates) (S);

3c. Kaudalansicht, GLC = Größte Länge des Humerus über das Caput gemessen.

Abb. 4: Radius; 4a. Dorsalansicht, Bd = Breite des distalen Gelenkkopfes, Bp = Breite des proximalen Gelenkkopfes, $\mathrm{GL}=$ Größte Länge, KD = Kleinster Durchmesser der Diaphyse (S); 4b. Proximaler Gelenkkopf von proximal, $\mathrm{BFp}=$ Breite der proximalen Gelenkfläche (S), Bp = Breite des proximalen Gelenkkopfes,

TFpm = Tiefe des medialen Teiles der proximalen Gelenkfläche (S); 4c und d. Distale Gelenkfläche von distal, $\mathrm{BFd}=$ Breite der distalen Gelenkfläche (S), BFdl = Breite des lateralen Abschnittes der distalen Gelenkfläche (S), $\mathrm{BFdm}=$ Breite des medialen Abschnittes der distalen Gelenkfläche (S).

Abb. 5: Metacarpus (Meßstrecken bei Metatarsus analog); 5a. Proximale Gelenkfläche von proximal, $\mathrm{Tp}=$ Tiefe der proximalen Gelenkfläche (S); 5 b. Volaransicht, Bd = Breite der distalen Gelenkrolle (S), Bdd = Breite unmittelbar proximal der distalen Gelenkrolle (S), Bp = Breite des proximalen Gelenkkopfes, $\mathrm{KD}=$ Kleinster Durchmesser der Diaphyse (S); 5c. Lateralansicht, GL = Größte Länge, $\mathrm{KT}=$ Kleinste Tiefe der Diaphyse (S).

Abb. 6: Femur; 6a. Kaudalansicht, Bd = Breite des distalen Gelenkkopfes, Bp = Breite des proximalen Gelenkkopfes, $\mathrm{KD}=$ Kleinste Breite der Diaphyse (S), Winkel $\alpha=$ Winkel zwischen der Gelenkfläche des Caput und dem Trochanter maior, Winkel $\beta=$ Öffnungswinkel der Fossa trochanterica; 6b. Distale Gelenkrolle von distal, BTd = Distale Breite der Trochlea femoris

(wegen des Knickes in der Lateralkante bei Bos primigenius wurde ca. $20 \mathrm{~mm}$ nach proximal verschoben gemessen) (S), BTp = Proximale Breite der Trochlea femoris (wegen häufiger Beschädigung des Proximalendes der Trochlea wurde ca. $20 \mathrm{~mm}$ nach distal versetzt gemessen) (S).

Abb. 7: Tibia; 7a. Dorsalansicht, Bd = Breite des distalen Gelenkkopfes, $\mathrm{Bp}=$ Breite des proximalen Gelenkkopfes, $\mathrm{GL}=$ Größte Länge, $\mathrm{KD}=$ Kleinster Durchmesser der Diaphyse (S); $7 \mathrm{~b}$. Medialansicht,

KT = Kleinste Tiefe der Diaphyse (S); 7c. Distale Gelenkfläche von distal, BFd = Breite der distalen Gelenkfläche (S), $\mathrm{BvF}=$ Mediolaterale Ausdehnung der plantaren Facette der Gelenkfläche für das Os malleolare (S), TFd = Tiefe der distalen Gelenkfläche (S). 

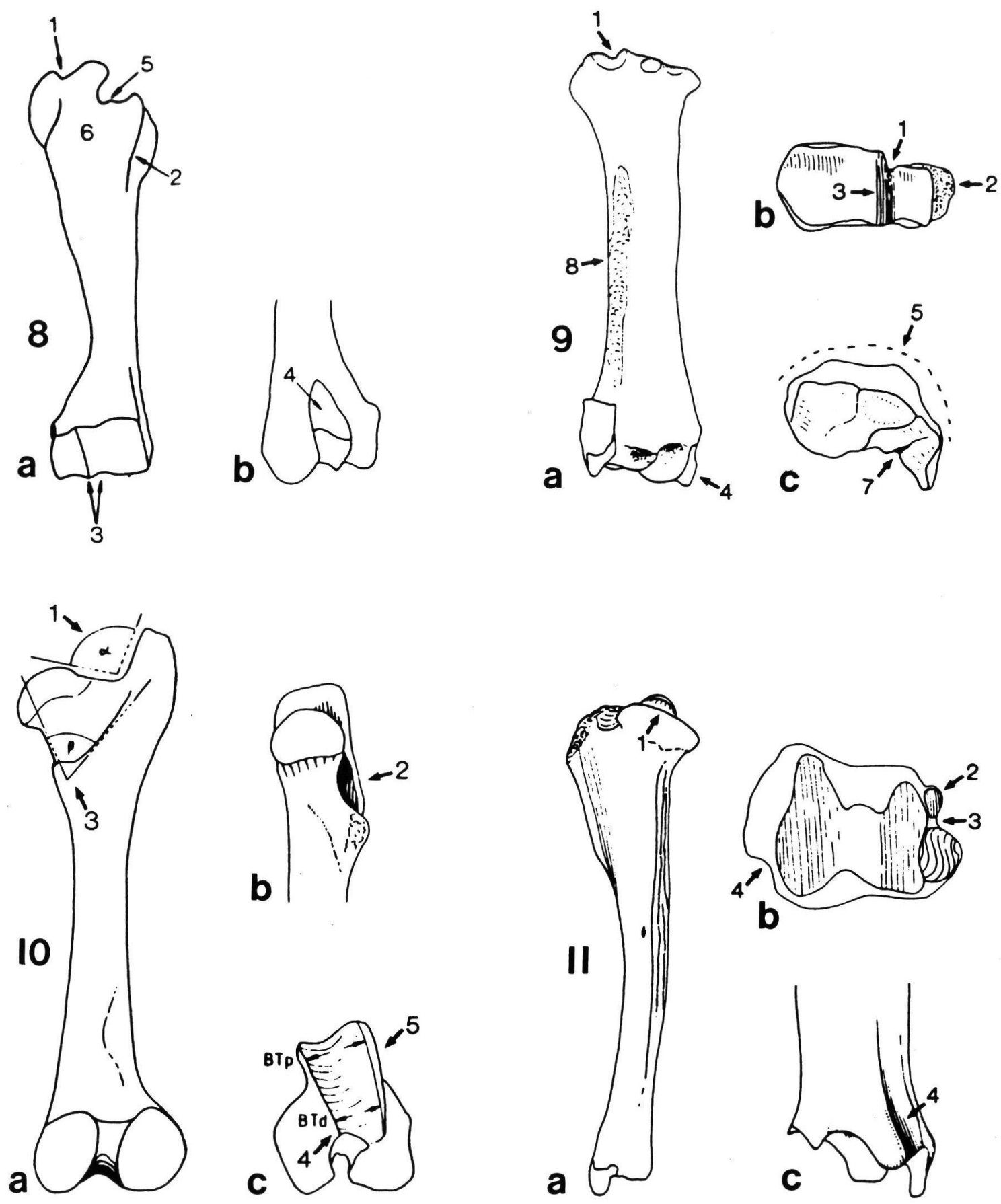

Abb. 8-11. Lage der diskutierten Unterscheidungsmerkmale an den Langknochen.

8. Humerus, 9. Radius. 10. Femur, 11. Tibia. 


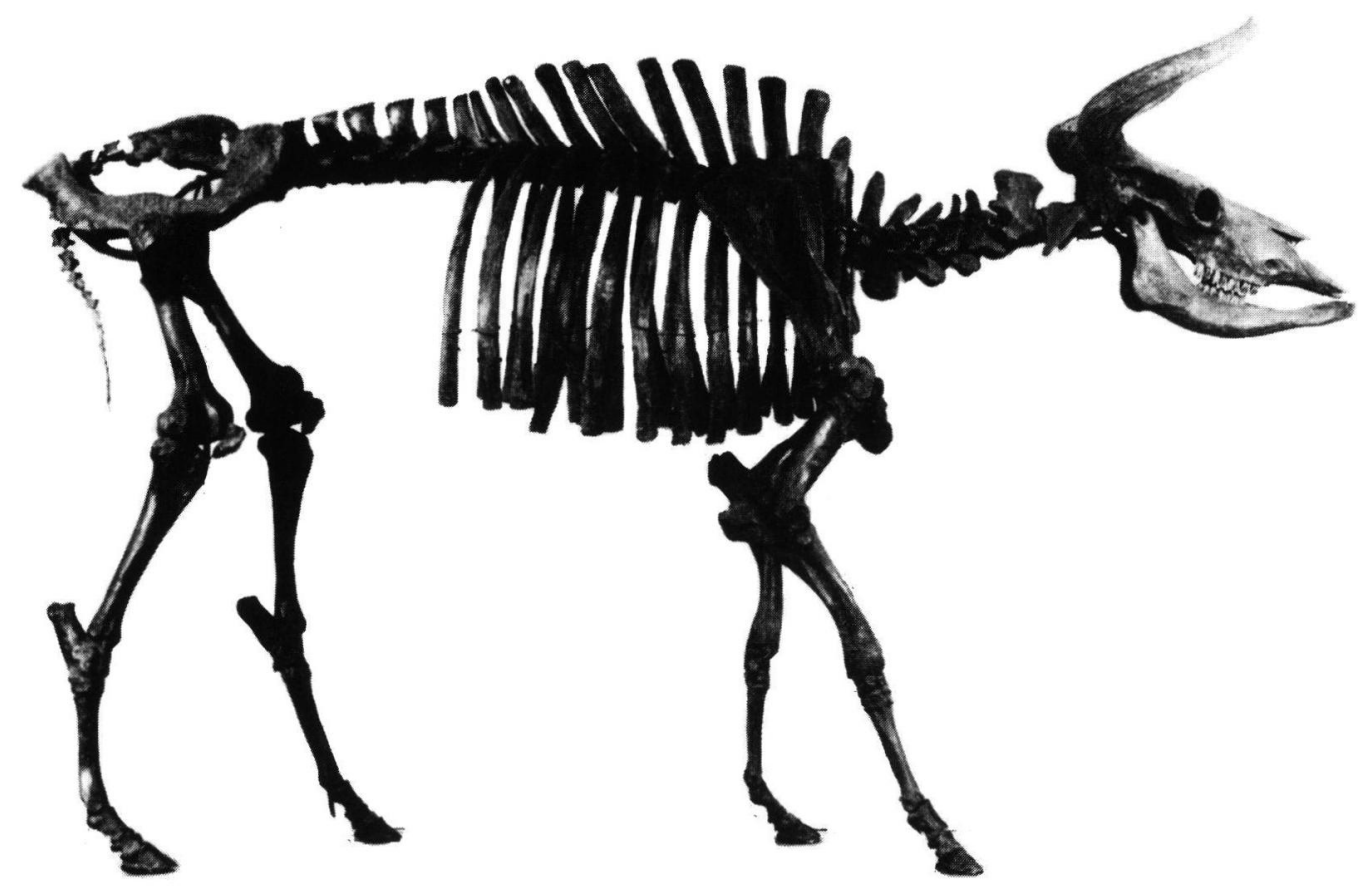

Abb. 12: Der Düsseldorfer Auerochse (Bos primigenius) in alter Montage (Bild: Archiv Löbbecke Museum).

Tab. 1: Meßwerte (in mm) am Schädel des Düsseldorfer und Braunschweiger Auerochsen.

Meßstrecke
Düsseldorfer

Auerochse
Braunschweiger

Auerochse
Gerade Entfernung d. Hornzapfenspitzen

Hornzapfenumfang an der Basis

Großer Hornzapfendurchmesser (oroaboral) an der Basis

Kleiner Hornzapfendurchmesser (dorsobasal) an der Basis

Außenlänge eines Hornzapfens

Länge der Nasalia

\section{8}

dex.

310

104

92

650

226
508

$\sin$.

310

105

92

680

220
717

dex. $\quad \sin$.

390

405

137

110

770

275

\subsection{Der Braunschweiger Auerochse}

Der Auerochse des Staatlichen Naturhistorischen Museums in Braunschweig wurde bereits von FIEDLER (1907) eingehend beschrieben. Das Skelett stammt nach FIEDLER (1907: 24) aus einem zwischen Alvesse und Vallstedt (ca. $8 \mathrm{~km}$ sw' Braunschweig) gelegenen Torfmoor und dürfte demnach holozänen Alters sein. Es soll hier nicht nur der Vollständigkeit halber erwähnt, sondern als weiterer Prüfstein der zu disku- tierenden Unterscheidungsmerkmale besprochen werden. Da das Skelett zur Zeit restauriert wird, bietet sich eine erneute Bestandsaufnahme der vorhandenen Knochen an.

Der Schädel mit beiden Hornzapfen ist bis auf die beiden in Gips ergänzten Prämaxillaria vollständig; die Zähne sind mäßig stark abgekaut.

Vom Achsenskelett ist die Wirbelsäule bis auf die Schwanzwirbel vollständig, die Rippen sind z. T. in 
Gips ergänzt. Am Schultergürtel ist die rechte Scapula vollständig erhalten, die linke nachgebildet. Bei beiden Humeri sind große Teile des proximalen Gelenkkopfes ergänzt; beide Radii sind mit Ulna erhalten. Carpalia, Metacarpalia und Phalangen sind vorhanden. Das Becken ist aus vielen Bruchstücken zusammengesetzt und der rechte äußere Sitzbeinast ist ergänzt. Bei beiden Femora sind proximale und distale Gelenkflächen nachgebildet. Die rechte Tibia ist vollständig, bei der linken ist der proximale Gelenkkopf teilweise ergänzt. Nach der frdl. Auskunft von Präparator STRAUSS fehlen einige Tarsalia. Die Metatarsalia sind unbeschädigt, die Phalangen bis auf das 1. Glied der Phalanx IV dex. vollständig vorhanden.

\section{Alter und Geschlecht}

Das bereits von FIEDLER (1907) als auffallend stark bezeichnete Auerochsenskelett hat nach der Form des Beckens und dem abgerundeten Pecten ossis pubis nach LEMPPENAU (1964) ein Hinweis auf ein männliches Becken - einem Bullen gehört. Nach dem Abnutzungsgrad der Zähne dürfte es sich um ein älteres Tier gehandelt haben, das ich aber nicht wie FIEDLER (1907) als senil bezeichnen möchte.

Die von FIEDLER (1907) noch nicht erwähnten Beschädigungen einiger Langknochen machen die Überprüfung wichtiger Bos/Bison-Unterscheidungsmerkmale leider unmöglich.

\subsection{Auerochsen-Teilskelett aus Erfelden}

Das Teilskelett mit bestimmbaren Schädelresten wurde im September 1987 in der Kiesgrube der Fa. Georg Fredder bei Erfelden in der nördlichen Oberrheinebene (ca. $10 \mathrm{~km}$ sw' Darmstadt) innerhalb weniger Tage mit dem Greifbagger im gleichen Grubenbereich zutage gefördert. Es wird im Hessischen Landesmuseum in Darmstadt unter den Nummern RS 3464 bis RS 3492 aufbewahrt.

Die Kiesgruben in der Umgebung von Erfelden erschließen Kiese und Sande des letzten Glazials sowie des letzten Interglazials (SCHWEISS 1988). Da Knochen nur in Zusammenhang mit dem industriellen Kiesabbau unter Wasser gewonnen werden können, ist eine genaue stratigraphische Einstufung klimatisch indifferenter Formen kaum möglich (KOENIGSWALD 1988).

\section{Umfang des Skelettes}

Vom Schädel ist die Hinterhauptsregion mit ca. $30 \mathrm{~cm}$ langen Stümpfen beider Hornzapfen, Stirnfläche, lin- ker Orbita und linkem Nasale im Verband erhalten; isoliert liegen neben einigen unbestimmbaren Bruchstücken der rechte Hinterhauptscondylus und ein Molar vor.

Vom Unterkiefer sind die linke Mandibel mit $\mathrm{P}_{4}$ (pathologisch abgenutzt) sowie ein kleines Fragment der rechten Mandibel mit $\mathrm{M}_{2}$ erhalten. Ober- und Unterkiefermolar sind mittelstark abgekaut. Vom Achsenskelett liegen der Epistropheus, ein weiterer Halswirbel, 2 Brustwirbel und Fragmente von mindestens 4 Rippen vor. An Elementen von Schultergürtel und Vorderextremitäten sind das proximokranial beschädigte linke Schulterblatt, beide Humeri (proximal bestoßen), der linke Radius mit distaler Hälfte der Ulna und das linke Metacarpale vorhanden.

Vom Becken liegen ein ca. $30 \mathrm{~cm}$ langes Fragment der linken und ein ca. $15 \mathrm{~cm}$ langes Fragment der rechten Darmbeinschaufel, ein ca. $20 \mathrm{~cm}$ langes Fragment des kaudalen Abschnittes der Ischium-Symphyse, sowie das rechte Acetabulum vor. Von den Extremitäten sind der Proximalteil des linken Femur, die rechte Tibia und der linke Calcaneus überliefert.

\section{Alter und Geschlecht}

Nach den Abkauungsgrad der wenigen vorhandenen Zähne dürfte es sich um ein Tier mittleren Alters gehandelt haben. Die erhaltenen Teile des Beckens sind für eine Geschlechtsbestimmung zu bruchstückhaft.

\subsection{Bison priscus-Skelett aus der Kiesgrube Groß-Rohrheim}

Das weitgehend vollständige Skelett (Abb. 13) wurde im Herbst 1985 in der Kiesgrube Groß-Rohrheim (ca. 20 km ssw' Darmstadt) innerhalb weniger Tage im gleichen Grubenbereich mit dem Saugbagger erfaßt (MARTIN 1987). Das den Ansatzpunkt des erhaltenen Hornzapfens weit überragende Hinterhaupt sowie die Krümmung des Hornzapfens ermöglichen eine eindeutige Bestimmung. Das Skelett wird im Hessischen Landesmuseum in Darmstadt unter den Nummern RS 3193 bis RS 3247 aufbewahrt.

In der Kiesgrube Groß-Rohrheim werden Kiese und Sande des letzten Interglazials sowie des letzten Glazials vom Abbau erfaßt (SCHWEISS 1988). Da BisonReste in interglazialen und glazialen Ablagerungen angetroffen werden, ist eine genaue stratigraphische Einstufung des Fundes nicht möglich. 


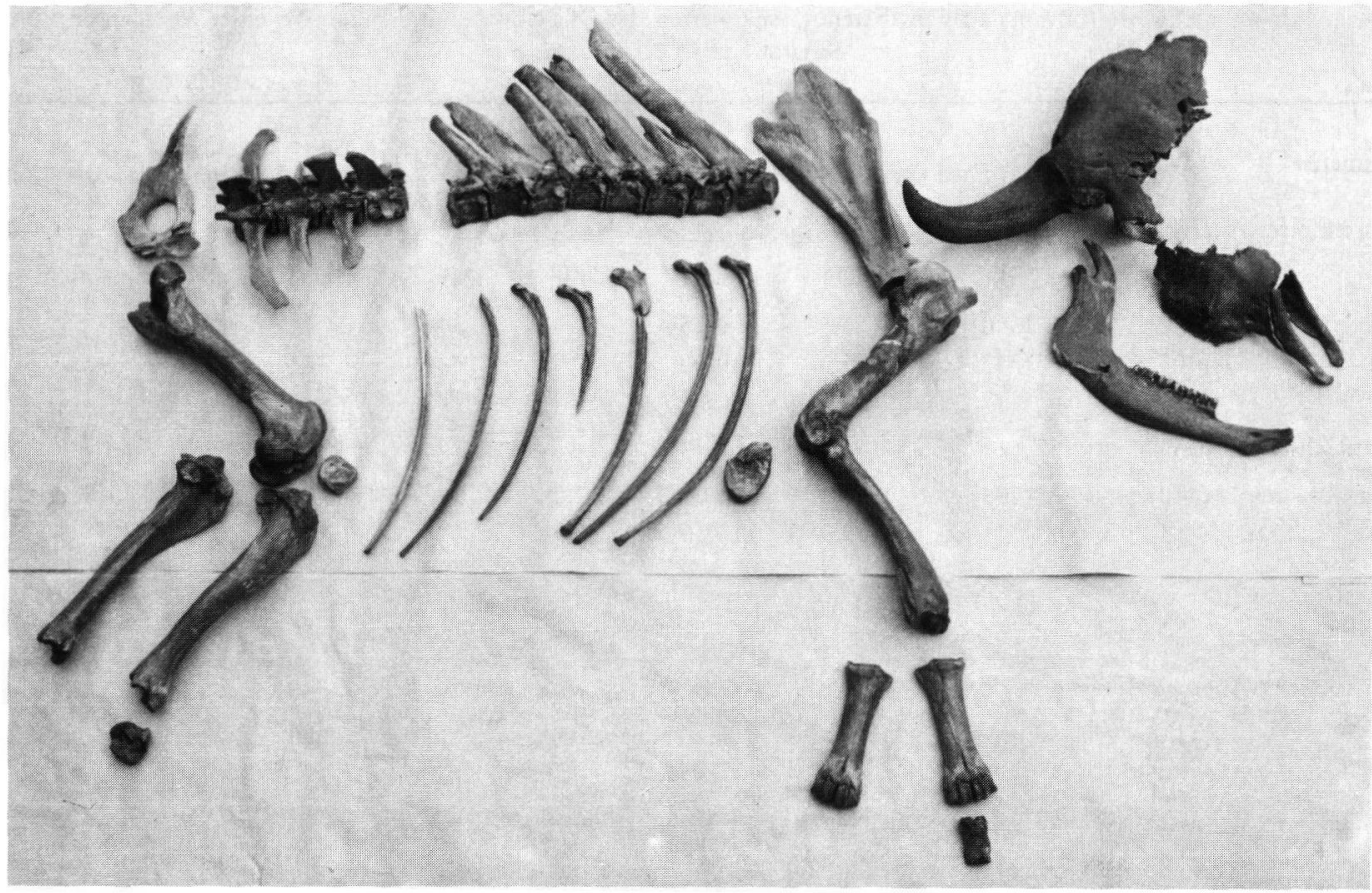

Abb. 13: Bison-Skelett aus der Kiesgrube Groß-Rohrheim in der nördlichen Oberrheinebene (HLMD-RS 3139 - RS 3247).

\section{Umfang des Skelettes}

Vom Schädel liegen ein Fragment des Neurocraniums mit linkem Hornzapfen, das rechte Maxillare mit Bezahnung, beide Prämaxillaria und die rechte Mandibel mit Bezahnung vor.

Vom Achsenskelett konnten 8 zusammengehörige Brustwirbel, 4 mehr oder weniger vollständige und Fragmente von mindestens 13 weiteren Rippen, ein ossifizierter Brustbeinknorpel und 4 zusammengehörige Lendenwirbel geborgen werden.

Vom Schultergürtel sind das linke Schulterblatt, dazu der rechte Humerus, der rechte Radius, beide Metacarpalia und ein Phalangenglied (Vorder- oder Hinterextremität) erhalten.

Die überlieferten Teile des Beckengürtels umfassen ein Beckenfragment (Symphysenbereich des Pubis mit linkem Acetabulum und Teilen des rechten Acetabulums), das linke Femur, die rechte Patella, die linke und rechte Tibia sowie das rechte Centrotarsale.

\section{Alter und Geschlecht}

Der kleine Hornzapfen, die tief abgekauten Molaren, die relativ zierlichen Knochen (mit verwachsenen Epi- physenfugen) und der ausgedünnte Vorderrand des Beckens (Pecten ossis pubis) - nach LEMPPENAU (1964) typisch für weibliche Becken - weisen auf eine ältere Kuh hin.

\subsection{Vergleichsmaterial aus dem Pleistozän von Großbritannien}

Von Bos primigenius aus Ilford (Essex) konnten 5 Humeri, 3 Radii, 2 Femora und 5 Tibiae und von Bison priscus aus Willment's Gravel Pit bei Isleworth (Middlesex) 11 Humeri, 19 Radii und 7 Tibiae im Britischen Museum untersucht werden. Die Bestimmung der Langknochen ergibt sich aus der Verteilung der Schädelfunde der beiden Boviden an den beiden Lokalitäten. Nach der freundlichen mdl. Mitteilung von A. CURRENT, London, fehlt Bos in Isleworth vollständig, während Bison häufig ist; umgekehrt verhält es sich mit Ilford, wo zahlreiche Auerochsenschädel, aber nur äußerst selten Bison-Schädel gefunden worden sind.

Auf der Mittelterrasse der Themse gelegen, wird Ilford in das jüngere Mittelpleistozän eingestuft (SUTCLIFFE 1985). Bei der Fundstelle Isleworth handelt es sich ebenfalls um Themse-Ablagerungen, die jedoch aus dem mittleren Abschnitt des letzten Glazials (43.000 a BP, COPE \& ANGUS 1975) stammen. 
Tab. 2: Meßwerte (in mm) an den Extremitätenknochen des Düsseldorfer und Braunschweiger Auerochsen; Schätzwerte in Klammern.

\section{Humerus}

Düsseldorfer Auerochse

$\begin{array}{lccccccc} & \text { GL } & \text { GLC } & \text { KD } & \text { BT } & \text { LAT } & \text { MHT } & \text { LHT } \\ \text { dex. } & (390) & (340) & 53,5 & 96,5 & 37,5 & * \star * & * \text { * } \\ \text { sin. } & (405) & (345) & 55,5 & 95,0 & 27,5 & * * & * *\end{array}$

Braunschweiger Auerochse

$\begin{array}{lccccccc}\text { dex. } & (440) & (398) & 59,0 & 109,0 & 32,0 & 64,5 & 59,0 \\ \text { sin. } & * \star * & * \star * & 59,5 & 107,0 & 30,5 & * \star * & * * *\end{array}$

\section{Radius}

Düsseldorfer Auerochse

\begin{tabular}{|c|c|c|c|c|c|c|c|c|c|}
\hline & GL & $\mathrm{Bp}$ & BFp & TFpm & $\mathrm{KD}$ & $\mathrm{Bd}$ & $\mathrm{BFd}$ & BFdm & BFdl \\
\hline dex. & $(360)$ & 111,0 & 98,0 & $* * *$ & 63,5 & 104,5 & $\star * *$ & $* * *$ & $\star \star *$ \\
\hline $\sin$. & 350,0 & 110,5 & 97,0 & $\star \star * *$ & 64,5 & 107,0 & $* * *$ & $\star \star * *$ & * * \\
\hline
\end{tabular}

Braunschweiger Auerochse

$\begin{array}{lccccccccc}\text { dex. } & 392,0 & 120,0 & 105,5 & 49,5 & 62,0 & 109,0 & 97,0 & 45,0 & 49,0 \\ \text { sin. } & * \star * & 121,0 & 109,0 & * \star * & 69,0 & * \star * & 95,0 & 43,5 & 47,0\end{array}$

\section{Metacarpus}

Düsseldorfer Auerochse

$\begin{array}{lllllll} & \text { GL } & \text { Bp } & \text { KD } & \text { KT } & \text { Bd } & \text { Bdd } \\ \text { dex. } & 245,0 & 80,5 & 48,5 & 30,5 & 79,0 & 72,5 \\ \text { sin. } & 245,0 & 80,0 & 48,5 & 30,5 & 79,0 & 73,0\end{array}$

Braunschweiger Auerochse dex. $\quad 259,0$ $\sin$. 83,0 52,0 31,5 $83,5 \quad 78,0$

$\begin{array}{lllll}81,5 & 51,0 & 32,0 & 82,5 & 78,5\end{array}$

\section{Tibia}

Braunschweiger Auerochse

$\begin{array}{lcccccccc} & \text { GL } & \text { Bp } & \text { KD } & \text { KT } & \text { Bd } & \text { BvF } & \text { BFd } & \text { TFd } \\ \text { dex. } & (485) & * * * & 59,5 & 40,5 & 92,0 & 19,5 & (58) & 61,0 \\ \text { sin. } & 488,0 & * \star * & 58,0 & 42,5 & 90,0 & 18,0 & (58) & 60,5\end{array}$

\section{Metatarsus}

Braunschweiger Auerochse

$\begin{array}{lccccccc} & \text { GL } & \text { Bp } & \text { KD } & \text { KT } & \text { Bd } & \text { Bdd } & \text { Tp } \\ \text { dex. } & 296,0 & 70,0 & 41,5 & 36,0 & 75,5 & 71,5 & 66,0 \\ \text { sin. } & 295,0 & 69,5 & 41,0 & 36,5 & 76,0 & 72,5 & 66,0\end{array}$


Im einzelnen wurden untersucht:

Bos primigenius von Ilford

Humerus: BMNH 45561 (dex.), BMNH 45562 (sin.), BMNH 45563 (sin.), BMNH 45564 (sin.), BMNH 48049 (dex.). Radius: BMNH 21299a (sin.), BMNH 45576 (dex.), BMNH 45579 (sin.). Femur: BMNH 45653 (dex.), BMNH 45663 (dex.). Tibia: BMNH 45672 (sin.), BMNH 45673 (dex.), BMNH 45674 (dex.), BMNH 45675 (dex.), BMNH 45680 (dex.).

Bison priscus von Isleworth (da die Knochen noch nicht inventarisiert sind, können hier nur die Feld-Nummern angegeben werden)

Humerus: Nr. 388 (sin.), Nr. 389 (sin.), Nr. 390 (dex.), Nr. 391 (dex.), Nr. 392 (dex.), Nr. 393 (dex.), Nr. 394 (dex.), Nr. 395 (dex.), Nr. 936 (dex.), Nr. 397 (dex.), Nr. 400 (sin.). Radius: Nr. 350 (dex.), Nr. 357 (dex.), Nr. 359 (dex.), Nr. 360 (dex.), Nr. 363 (dex.), Nr. 364 (sin.), Nr. 366 (sin.), Nr. 367 (sin.), Nr. 369 (dex.), Nr. 370 (dex.), Nr. 371 , Nr. 372 (sin.), Nr. 373 (sin.), Nr. 374 (sin.), Nr. 375 (sin.), Nr. 377 (sin.), Nr. 380 (sin.), Nr. 384 (sin.), o. Nr. (sin.). Femur: - - Tibia: Nr. 265 (dex.), Nr. 267 (dex.), Nr. 276 (sin.), Nr. 277 (sin.), Nr. 278 (sin.), Nr. 279 (sin.), Nr. 280 (sin.).

\subsection{Vergleichsmaterial von Bison div. sp. aus Nordamerika}

Nach ANDERSON (1984) erreicht Bison priscus Alaska von Eurasien her im frühen Rancholabrean (entspricht etwa dem späten Mittel- und Jungpleistozän). WILSON (1974: 142) unterscheidet in der weiteren Entwicklung zwischen einem nördlichen und südlichen Phänotyp. Die Vertreter des nördlichen Phänotyps entwickeln sich nach WILSON (1974) über Bison priscus alaskensis und Bison priscus crassicornis zu Bison bison athabascae und Bison bison occidentalis. Der südliche Zweig brachte dagegen über den langhornigen Bison priscus latifrons und Bison priscus alleni die Formen Bison bison antiquus und Bison bison bison hervor. WILSON (1974) nennt einen Genfluß zwischen beiden Populationen wahrscheinlich. Dagegen unterscheidet MCDONALD (1981) bei den jungpleistozänen bzw. rezenten Formen zwischen 2 Arten: Bison antiquus (mit den Unterarten B. a. antiquus und B. $a$. occidentalis) und Bison bison (mit $B . b$. bison und $B$. b. athabascae). In der vorliegenden Arbeit wird die Klassifikation von MCDONALD benutzt.

$\mathrm{Da}$ die nordamerikanischen Bisons im wesentlichen nach der Hornform und Horngröße unterschieden werden, ist eine artliche Zuweisung von isolierten Langknochen kaum möglich; werden im folgenden trotzdem Artnamen genannt, sind sie aus den Unterlagen der jeweiligen Sammlungen entnommen, oder es handelt sich um Material von Lokalitäten, die nur eine Bison-Art geliefert haben.
Bison antiquus occidentalis und Bison sp. von der Natural Trap Cave (NTC), Wyoming

Bei der Natural Trap Cave handelt es sich um einen ca. $20 \mathrm{~m}$ tiefen Karsthohlraum in unterkarbonischen Kalken in den Bighorn Mountains im nördlichen Wyoming. In diese natürliche Falle, die nur in ihrer Decke eine Öffnung aufweist, sind nach radiometrischen Datierungen von Aschelagen (GILBERT \& MARTIN 1984) seit der Sangamon-Warmzeit (entspricht wohl dem Eem) bis in heutige Zeit unzählige Tiere gestürzt (MARTIN \& GILBERT 1978a); ihre Überreste bildeten im Laufe der Zeit eine mindestens $3 \mathrm{~m}$ mächtige Knochenpackung (MARTIN et al. 1977). Neben Klein- und anderen Großsäugern, wie z.B. Wolf (Canis sp.), amerikanischem Löwen (Panthera atrox), Pferd (Equus sp.), Kamel (Camelops sp.) und einem Bergschaf (Ovis catclawensis) wurden in der Höhle zahlreiche Überreste von Bison sp. (MARTIN \& GILBERT 1978 b) gefunden. Die meisten Knochen haben nach ${ }^{14} \mathrm{C}$-Datierungen ein Alter von 21.000 bis 12.000 Jahren.

Humerus: KUMVP-NTC 25980 (dex.), KUMVP-NTC 51275 (dex.); Radius: KUMVP-NTC 47955 (sin.), KUMVP-NTC 48916 (dex.); Femur: KUMVP-NTC 82210 (dex.); Tibia: KUMVP-NTC 26498 (sin.), KUMVP-NTC 42750 (sin.), KUMVP-NTC 42887 (dex.), KUMVP-NTC 44361 (sin.), KUMVP-NTC 45338 (dex.), KUMVP-NTC 57017 (dex.).

Bison sp. aus den Kansas River Sand bars

Die Kies- und Sandbänke des Kansas-River im Ostteil des Bundesstaates Kansas liefern zahlreiche Knochen von Wirbeltieren des Jungpleistozäns bis Holozäns; dabei machen unter den Großsäugern Reste von Bison div. sp. einen Großteil der Funde aus (MARTIN et al. 1979).

Humerus: KUMVP 82882 (sin.), KUMVP 88276 (dex.), KUMVP 88289 (sin.), KUMVP 88550 (dex.); Radius: - -; Femur: - -; Tibia: KUMVP 59485 (sin.), KUMVP 59487 (dex.), KUMVP 63757 (dex.), KUMVP 88277 (dex.), KUMVP 82281 (dex.).

Bison antiquus occidentalis und Bison sp. vom 12-Mile Creek

In den Kiesbänken des 12-Mile Creek im Westen des Bundestaates Kansas wurde Ende letzten Jahrhunderts eine Ansammlung fossiler Bison-Skelette entdeckt. Eine Pfeilspitze und Schlachtspuren an einigen Knochen lassen darauf schließen, daß die Bisons vom Frühmenschen auf der Jagd getötet wurden („Bison Kill"). Zwei Radiokarbondatierungen einer BisonTibia ergaben ein gemitteltes Alter von 10.300 a BP (ROGERS \& MARTIN 1984).

Humerus: KUMVP 102704 (dex.), KUMVP 102706 (sin.), KUMVP 102705 (sin.), KUMVP o. Nr. (sin.). Radius: KUMVP 102709 (dex.), KUMVP 102708 (dex.), Nr. 3190 
(sin.), NR. 3190 YY; Femur: - -; Tibia: KUMVP 102713 (sin.), KUMVP 102716 (dex.), KUMVP 102712 (dex.), KUMVP 102711 (dex.), KUMVP 102715 (dex.), KUMVP 102714 (dex.).

Darüber hinaus stand ein holozänes Bison-Skelett (Bison bison) aus den Big Snowy Mountains (Montana) (Loc.; KU Mt 41) zur Verfügung (KUMPV 23002).

\section{Rancho La Brea (Los Angeles)}

In den Asphaltsümpfen von Rancho La Brea wurde eine vielfältige Wirbeltierfauna des Jungpleistozäns und Holozäns (Rancholabrean) konserviert (STOCK 1972). Die Bison-Reste stammen fast ausnahmslos von Bison antiquus, nur vereinzelte Knochen wurden einem sehr großen Bison, B. ?latifrons, zugeordnet (MILLER \& BROTHERSON 1979).

Soweit nur die Feldnummern angegeben werden können, sind die in Abb. 14-17 verwendeten Kurz-Nummern (mit **) beigefügt.

Humerus: Nr. 3/E4, $61 / 2$ (dex.) $={ }^{* *} 1$; Nr. 4, B-5, 12-15 (dex.) $={ }^{\star \star} 2$; Nr. pit 4, F 4-5, $141 / 2(\sin )=.{ }^{\star \star} 3$; Nr. E, $3 / 4,63 / 4(\sin )=.{ }^{*} 4 ;$ Nr. $3 / \mathrm{D} 2,9$ (dex. $)={ }^{* *} 6$; LACM Y 6656; LACM Y 6662 (sin.); LACM Y 6663 (sin.); LACM HCV 6022. Radius: Nr. 13/9-11 (dex.) $={ }^{\star \star} 7$; Nr. $13 /$ C11, 15 (dex.) $={ }^{\star \star} 8$; Nr. 4, C3 +4, $111 / 269$ (dex.) $={ }^{* *} 9$; Nr. 60/ $91 / 2-10^{\prime}, 376(\sin )=.{ }^{* *} 10 ;$ Nr. 67 (sin.) $={ }^{\star \star} 11 ;$ Nr. $61 / 81 / 2-10^{\prime}$ (dex.) $={ }^{\star *} 12 ; \mathrm{Nr} .61 / \mathrm{E} 10$, 13 (dex.) $={ }^{* \star} 13 ;$ Nr. $60 /$ E-10, $12-15,405$ (dex. $)={ }^{* * 14}$; Nr. 61/F 15, 12-16 (dex.) $={ }^{\star \star} 15$; Nr. 3, F-2, 9' (dex.) $={ }^{*} 16 ;$ Nr. $4, \mathrm{E} 4+5,16,2 \times 9(\sin )=.{ }^{* *} 17$; LACM Y 6697 (sin.). Femur: Nr. 3, E-2,9 (sin.) = * 18 ; Nr. 3, D-4, $91 / 2(\sin )=.{ }^{* *} 19 ; \mathrm{Nr} .4 / \mathrm{F} 4+5,9$ (dex. $)={ }^{* *} 20$; Nr. 131 F11, 11.5 (dex.) $={ }^{*} 21 ;$ Nr. $16 / 3-6$ (dex. $)={ }^{*} 22$; LACM Y 6779 (sin.); LACM Y 6782 (sin.); LACM Y 6783 (dex.). Tibia: Nr. 4/F-4 +5, 11-12, 236 (sin) $={ }^{\star \star} 23$; Nr. 4/A-5, 11' (dex.) = "*24; LACM Y 6790 (dex.); LACM Y 6791 (dex.); LACM Y 6802 (sin.); LACM HC 6016 (sin.).

\section{Merkmalsausprägung an den Extremitätenknochen}

Zur Darstellung der Merkmalszustände an den einzelnen Knochen hat sich eine graphische Darstellung (Abb. 14 bis 17) bewährt, die einen übersichtlichen Vergleich der Merkmalskombinationen auch bei größeren Materialmengen erlaubt. Jedes Merkmal wird durch ein schwarzes Rechteck vertreten. Liegt das schwarze Rechteck über der Grundlinie, ist das betreffende Merkmal typisch für Bos primigenius ausge- prägt, befindet es sich unter der Grundlinie, liegt das Merkmal in Bison-typischer Ausprägung vor. Intermediäre Merkmalszustände werden durch ein auf der Grundlinie liegendes Rechteck angezeigt. Konnte ein Merkmal z. B. wegen Beschädigung nicht beurteilt werden, bleibt der Platz frei. Eine eingehende Erläuterung der einzelnen Bestimmungsmerkmale wurde in einer früheren Arbeit vorgenommen (MARTIN 1987).

\subsection{Humerus}

Am Humerus wurden die folgenden Merkmale (Abb. 8) zur Unterscheidung von Bos primigenius und Bison priscus diskutiert. Die Nummern der Merkmale entsprechen denen in Abb. 14.

1. Eine Einkerbung im Tuberculum maius fehlt bei Bos und ist bei Bison vorhanden.

2. Der Übergang der Diaphyse in das Tuberculum minus erfolgt bei Bos abrupt und bei Bison allmählich.

3. Das Relief von Führungsgrat und Rinne der Trochlea humeri ist bei Bos kräftig und bei Bison schwach ausgeprägt.

4. Die Fossa olecrani ist bei Bos breit-kastenförmig und bei Bison schmal.

5. Ein Tuberculum intermedium soll bei Bos fehlen und bei Bison vorhanden sein.

6. Der proximale Gelenkkopf ist bei Bos breiter als bei Bison.

Die Verteilung der Merkmalsmuster an den Humeri kann der Abb. 14 entnommen werden. Dort erscheint die Zuverlässigkeit von Merkmal 1 (Einkerbung im Tuberculum maius) abgeschwächt. Offensichtlich spielen hier altersbedingte Veränderungen doch eine größere Rolle als zunächst angenommen.

Merkmal 2 (Übergang der Diaphyse in das Tuberculum minus) wird als gut brauchbar bestätigt. Als weitgehend zuverlässig erwies sich Merkmal 3 (Führungsgrat und Rinne der Trochlea humeri); Abweichungen traten fast ausschließlich bei zierlichen Bison-Humeri auf. Dieses Merkmal ist auch deshalb wichtig, weil es das verläßlichste am besonders häufig erhaltenen distalen Gelenkende ist. Die Bedeutung von Merkmal 4 (Form der Fossa olecrani) wird geringer, Merkmal 5 (Tuberculum intermedium) erscheint wie bisher wenig aussagekräftig. Merkmal 6 (Form des proximalen

Abb. 14: Verteilungsmuster der Merkmalsausprägungen am Humerus.

Feldnummern sind durch ein * gekennzeichnet oder durch Nummern mit * ${ }^{*}$ vertreten (Zuordnung siehe 3.5 ); weitere Erklärung im Text. 

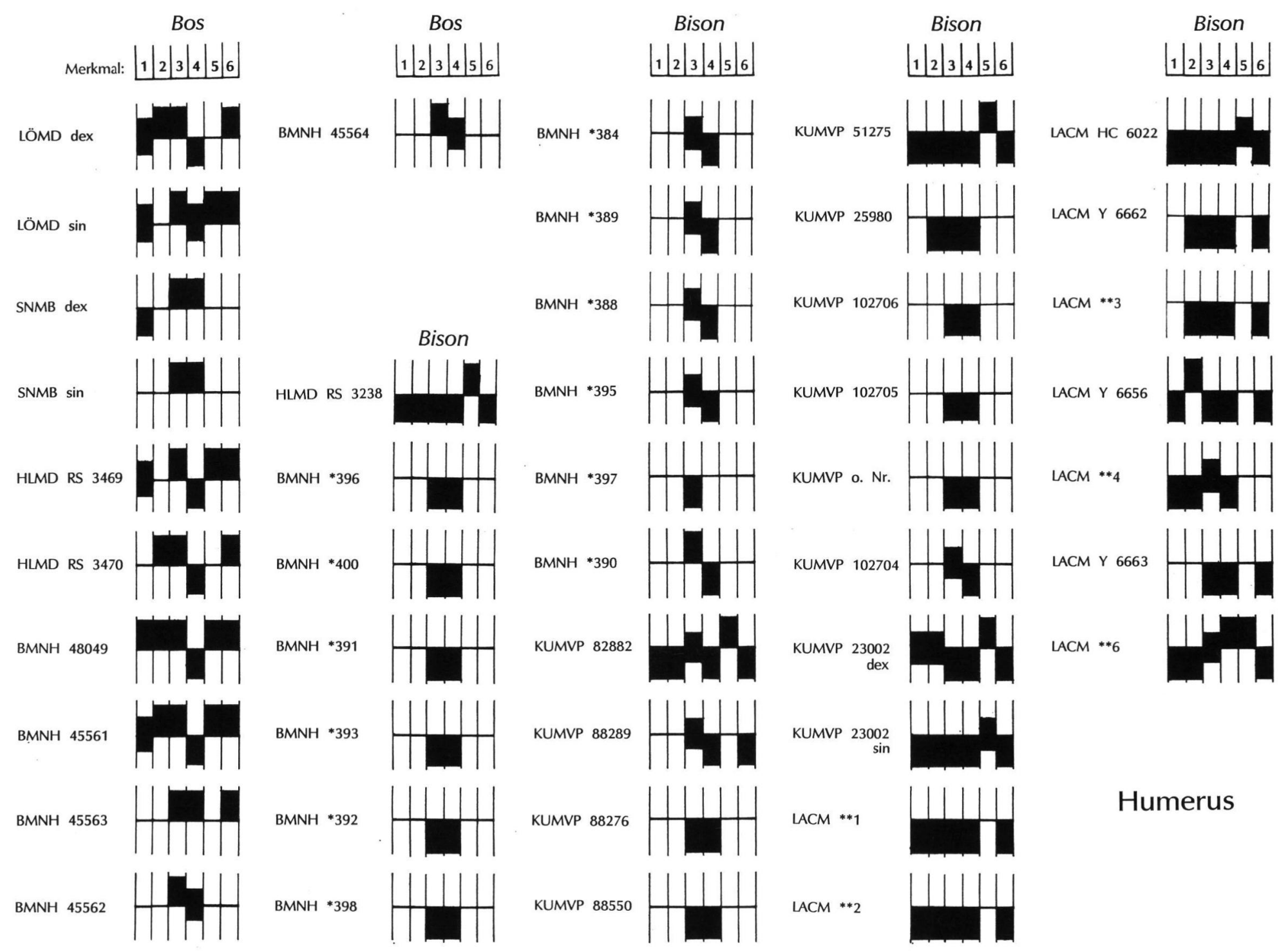
Gelenkkopfes) wird bestätigt, ist aber nur bei den wenigen Stücken, bei denen der dünnwandige Proximalteil nicht zu stark beschädigt ist, beurteilbar.

\subsection{Radius}

Am Radius wurden folgende Unterscheidungsmerkmale diskutiert (Abb. 9). Die Nummern der Merkmale entsprechen denen in $\mathrm{Abb} .15$.

1. Die Verbindungsfläche zur Ulna an der Volarseite der proximalen Gelenkfläche ist bei Bos tief eingekerbt, und von der Ulna greift ein Zapfen in die Kerbe hinein. Bei Bison ist die Verbindungsfläche nicht eingekerbt sondern glatt.

2. Der laterale Bandhöcker am Caput radii springt bei Bos kräftig vor und ist bei Bison wenig prominent.

3. Das Relief der proximalen Gelenkfläche ist bei Bos kräftig und bei Bison seicht.

4. Die Facette für das Os radiale verschmälert sich bei Bos nach kaudal stark, bei Bison jedoch kaum.

5. Die distale Gelenkfläche ist bei Bos stark und bei Bison schwach gekrümmt.

6. Der distale Gelenkflächenindex (n. BIBIKOVA 1958; $\frac{\text { BFdm*100 }}{\text { BFdl }}$ ) erreicht bei Bos höchstens 95 und geht bei Bison nicht unter 101.

7. Bei Bos tritt in der Facette für das Os ulnare eine Kerbe auf, bei Bison fehlt sie.

8. Die Verbindung der Diaphysen von Radius und Ulna ist bei Bos vollständig verknöchert, bei Bison weitgehend knorpelig.

Zur Verteilung der Merkmalsmuster an den Radien siehe Abb. 15. Merkmal 1 (Verbindungsstelle zur Ulna), 2 (Lateraler Bandhöcker am Caput radii) und 3 (Relief der proximalen Gelenkfläche) werden als wichtige Bestimmungsmerkmale bestätigt. Merkmal 4 (Facette für das Os radiale) erscheint, besonders beim Material aus Großbritannien, weniger zuverlässig. Merkmal 5 (Krümmung der distalen Gelenkfläche) wird bestätigt. Die Merkmale 7 (Kerbe in der Facette für das Os ulnare) und 8 (Verbindung der Diaphysen von Radius und Ulna) erwiesen sich auch hier als unbrauchbar, da sie stark altersabhängig sind. Die neuen Untersuchungen ergaben eine Verringerung der Bestimmungsmöglichkeit distaler Radius-Bruchstücke.

\subsection{Femur}

Am Femur wurden folgende Unterscheidungsmerkmale diskutiert (Abb. 10). Die Nummern der Merkmale entsprechen denen in Abb. 16.

1. Der Trochanter maior steigt bei Bos steil (Winkel zwischen Caput femoris und Trochanter maior ca. $90^{\circ}$ ) und bei Bison flacher (Winkel ca. $100-110^{\circ}$ ) an.

2. Die Fossa trochanterica ist bei Bos weit offen und bei Bison leicht verengt.

3. Der Winkel an der Fossa trochanterica liegt bei Bos über $65^{\circ}$ und erreicht bei Bison maximal $62^{\circ}$.

4. Der laterale Begrenzungsgrat der Facies patellaris hat bei Bos eine distale Einknickung, die bei Bison fehlt.

Der Trochlea-Index ( $\left.\frac{\mathrm{BTd} * 100}{\mathrm{BTp}}\right)$ liegt bei Bos über 92 und erreicht bei Bison maximal 89.

Die Verteilung der Merkmalsmuster an den Femora kann Abb. 16 entnommen werden. Merkmal 1 (Anstieg des Trochanter maior) war für eine erneute Beurteilung zu selten erhalten, was auch für Merkmal 3 (Winkel an der Fossa trochanterica) gilt. Merkmal 2 (Öffnung der Fossa trochanterica) war bei Bos primigenius zuverlässig, bei Bison allerdings wenig konstant (z. B. Material von Rancho La Brea). Merkmal 4 (Lateraler Begrenzungsgrat der Facies patellaris) erwies sich weiterhin als zuverlässig. Auch der TrochleaIndex kann weiterhin als gutes Bestimmungsmerkmal gelten.

\subsection{Tibia}

An der Tibia wurden folgende Unterscheidungsmerkmale diskutiert (Abb. 11). Die Nummern der Merkmale entsprechen denen in Abb. 17.

1. Der laterale Rand des Condylus lateralis ist bei Bos schwach und bei Bison stark gekrümmt.

2. Die dorsale Gelenkfläche für das Os malleolare ist bei Bos flach und steht senkrecht zur Längsachse des Knochens; bei Bison ist sie geknickt bzw. steht schräg zur Längsachse des Knochens.

3. Der Einschnitt für den Zapfen des Os malleolare ist bei Bos seicht und bei Bison tief.

4. Die Rinne für den Musculus flexor digitalis ist bei Bos tief und bei Bison seicht. 

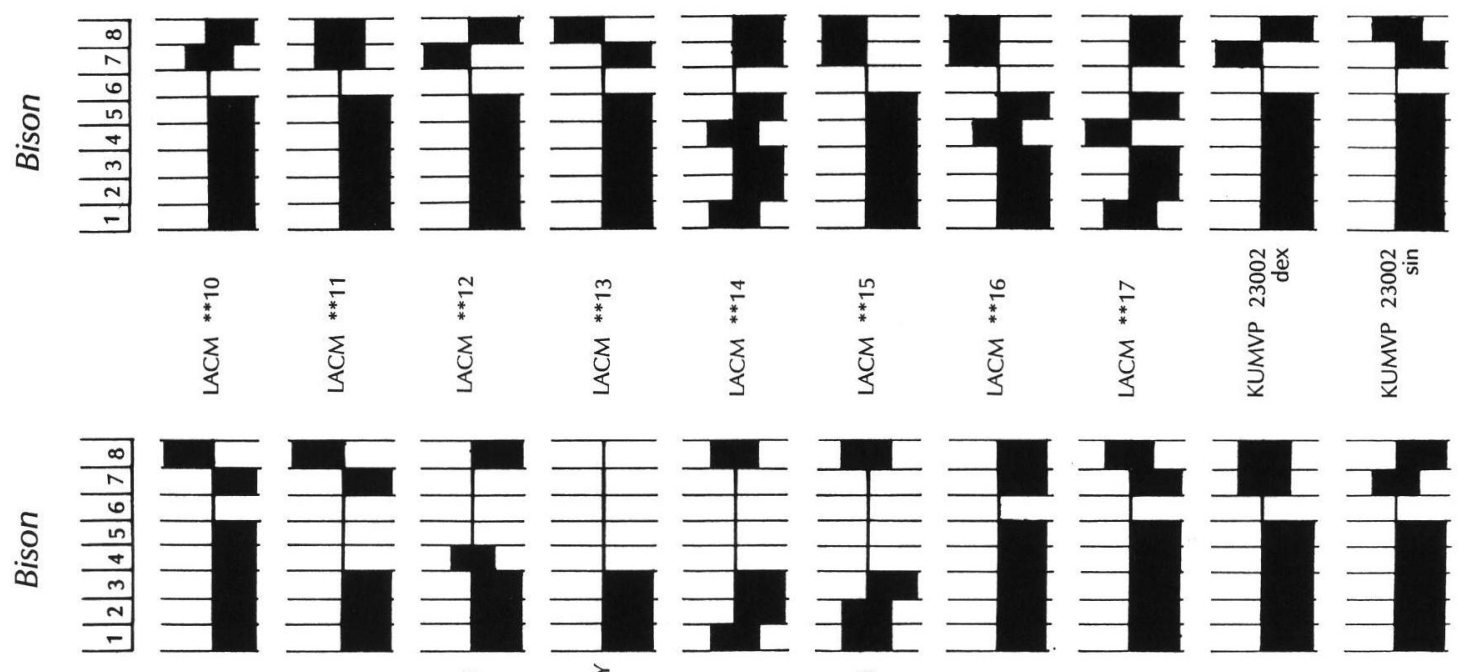

高

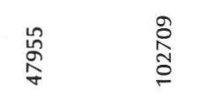

章

衰

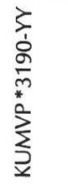

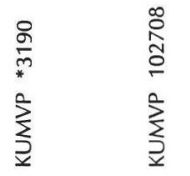

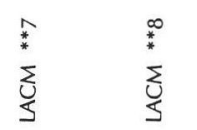

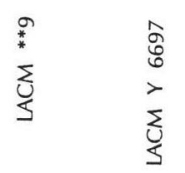

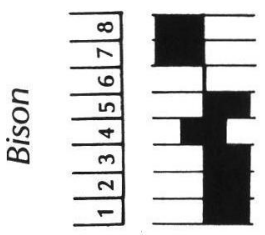
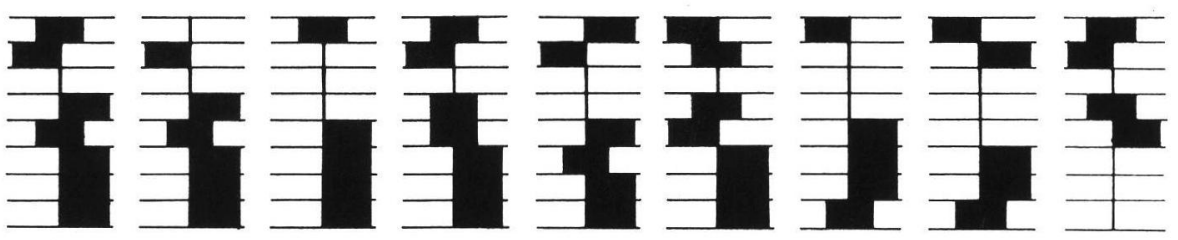

$\stackrel{+}{\infty}$
$\stackrel{1}{\infty}$
$\sum_{\infty}^{\frac{1}{Z}}$

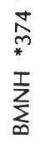

管

$\begin{array}{ll}\underset{\infty}{*} & \stackrel{0}{\infty} \\ \sum_{\infty}^{T} & \sum_{\infty}^{T}\end{array}$

令
$\sum_{\infty}^{*}$
T

$W_{0}^{\infty}$
$\sum_{\infty}^{T}$

웅

$\sum_{\infty}^{\frac{T}{2}}$
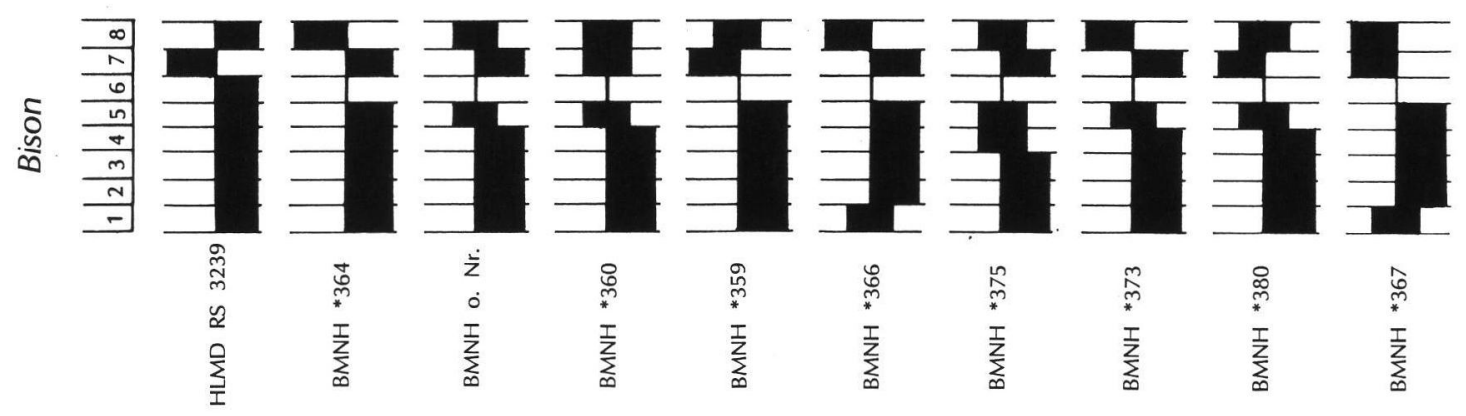

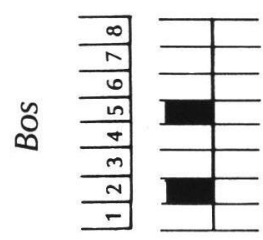
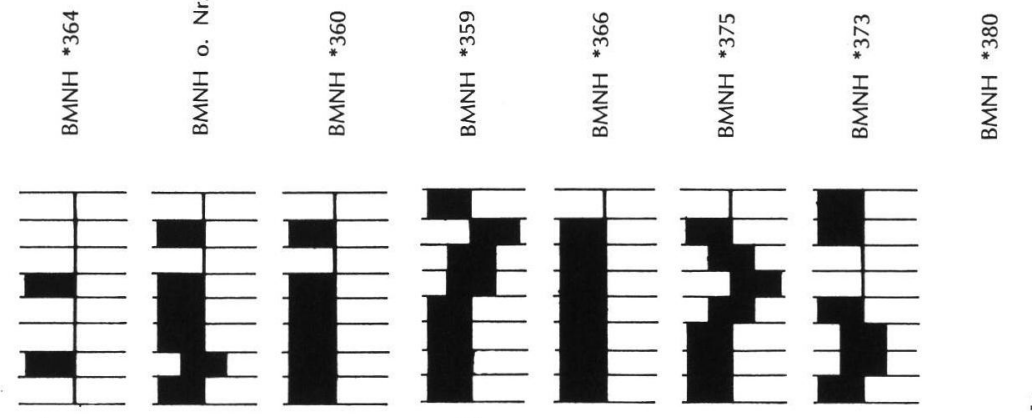

$$
\text { 点 }
$$
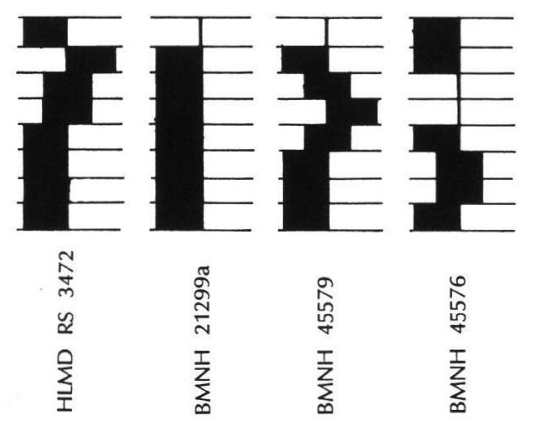


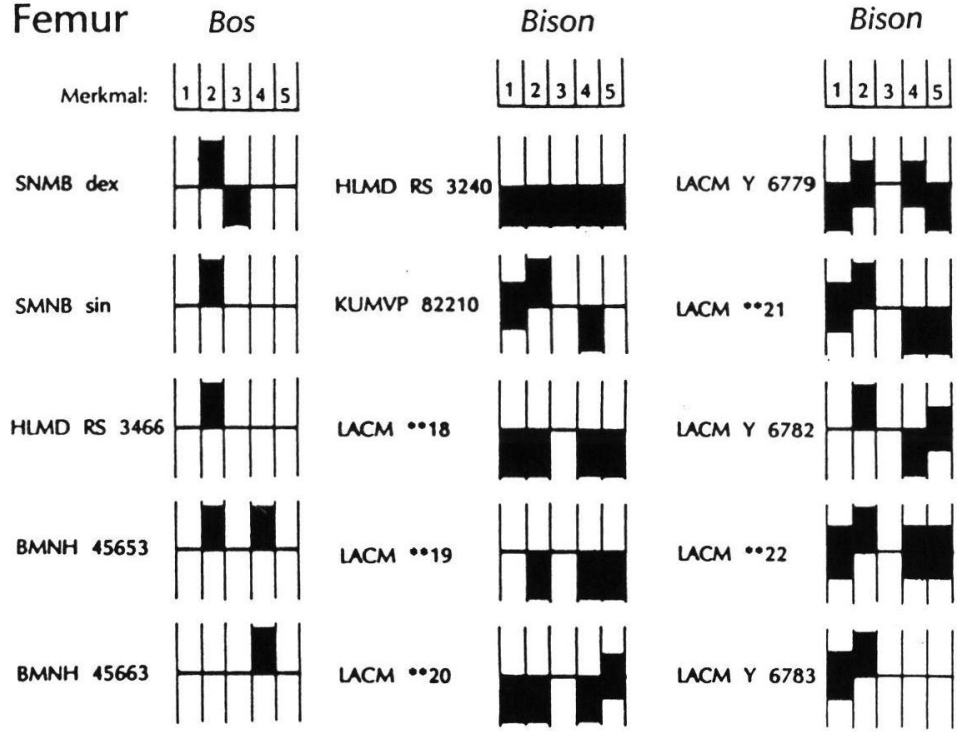

\begin{abstract}
Abb. 16: Verteilungsmuster der Merkmalsausprägungen am Femur. Feldnummern sind durch ein * gekennzeichnet oder durch Nummern mit ** vertreten (Zuordnung siehe 3.5); weitere Erklärung im Text.
\end{abstract}

Die Verteilung der Merkmalsmuster an den Tibien ist in Abb. 17 dargestellt. Merkmal 1 (lateraler Rand des Condylus lateralis) hat sich in den wenigen Fällen, in denen es erhalten war, bestätigt. Merkmal 2 (Form und Stellung der dorsalen Gelenkfläche für das Os malleolare) hat sich, besonders beim Bos-Material von Ilford, als weniger zuverlässig erwiesen; dasselbe gilt bei Bison für Merkmal 3 (Einschnitt für den Zapfen des Os malleolare). Ausnahmslos zuverlässig war Merkmal 4 (Grube für den Musculus flexor digitalis); es kann als einziges der diskutierten Unterscheidungsmerkmale beinahe als Schlüsselmerkmal verwendet werden.

\section{Vergleich mit Bison div. sp.}

\section{aus dem nordamerikanischen Pleistozän}

Die natürlichen Verbreitungsgebiete der amerikanischen Bisons und von Bos primigenius haben sich nicht überschnitten. Deshalb scheint eine Überprüfung, ob Bison bison und Bison antiquus sich vom Auerochsen osteologisch unterscheiden, zunächst ohne großen praktischen Wert zu sein. Zieht man jedoch das Problem einer Vermischung (sub)fossiler Bison-Knochen mit den Knochen eingeführter Rinder (Bos primigenius f. taurus) z. B. in indianischen Jagdstationen in Betracht, wird die Bedeutung einer Unterscheidungsmöglichkeit offensichtlich (OLSEN 1960). Außerdem ist es reizvoll, zu überprüfen, ob Merkmale, die Bison priscus von Bos primigenius unterscheiden, innerhalb der Gattung Bison konstant bleiben.

An den Langknochen von Bison antiquus und Bison bison sind die an Bison priscus erarbeiteten Unter- scheidungsmerkmale generell brauchbar. Bei einigen Merkmalen sind jedoch Abweichungen zu beobachten. So fällt z.B. am Humerus auf, daß das an sich sehr zuverlässige Merkmal 3 (Führungsgrat und Rinne der Trochlea humeri) bei einigen Stücken weniger konstant erscheint. Hierbei handelt es sich durchweg um sehr zierliche Knochen. Das gleiche Phänomen, nämlich daß bei kleinen Humeri die distale Gelenkrolle ein schärferes Relief als bei größeren zeigt, wurde auch an Material aus den jungpleistozänen Rheinschottern beobachtet (MARTIN 1987). Dieses Merkmal sollte deshalb nur bei Knochen, die in der normalen Größenordnung von Bison priscus liegen, zur Bestimmung herangezogen werden.

Der Vergleich hat gezeigt, daß die Ausprägung der betreffenden Merkmale beim nordamerikanischen $B i$ son antiquus und $B$. bison weitgehend mit der beim europäischen Bison priscus übereinstimmt.

\section{Zusammenfassung}

Die an Bovidenknochen aus den Rheinschottern ausgearbeiteten Unterscheidungsmerkmale für Bos primigenius und Bison priscus (MARTIN 1987) werden durch sicher bestimmte Skelettfunde bestätigt. Umgekehrt erwiesen sich verschiedene früher diskutierte Merkmale, die bereits am Rheinschottermaterial wegen zu starker Variabilität oder Altersabhängigkeit wenig brauchbar waren, auch hier als nutzlos. Die Rinne für den Musculus flexor digitalis am Distalende der Tibia trat am untersuchten Material jeweils in typischer Ausprägung auf und kann deshalb beinahe als Schlüsselmerkmal gewertet werden. 
Tibia Bos

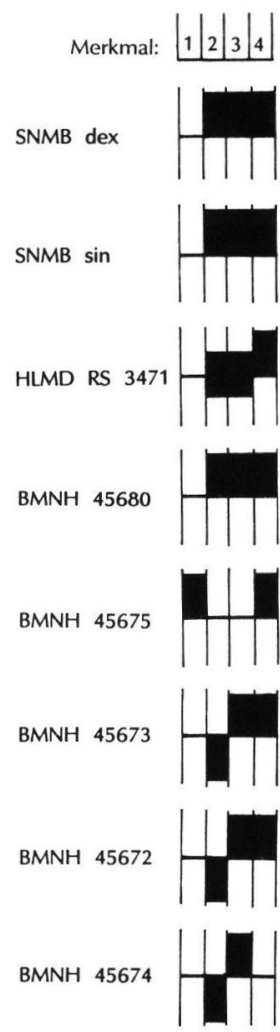

Bison

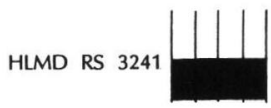

HLMD RS 3242||$\downarrow||$
Bison
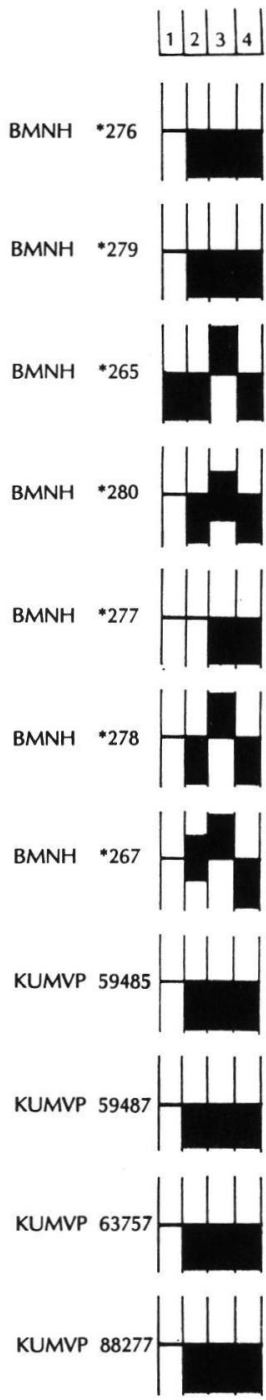

Bison
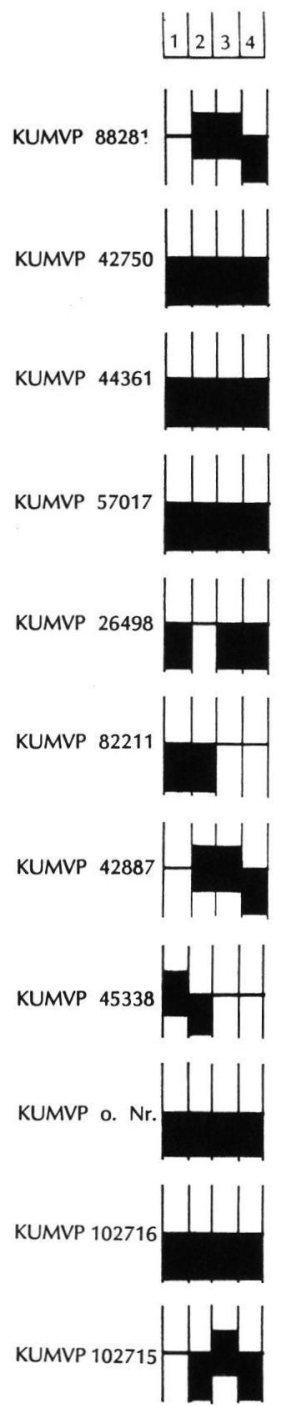
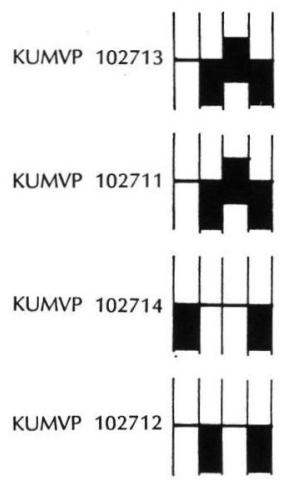

LACM Y 6790
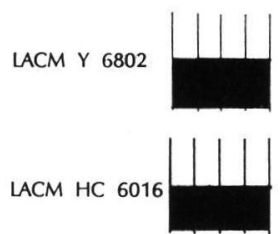

LACM $* * 23$
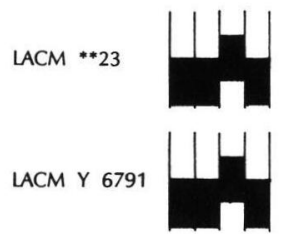

LACM $^{* *} 24$

Bison
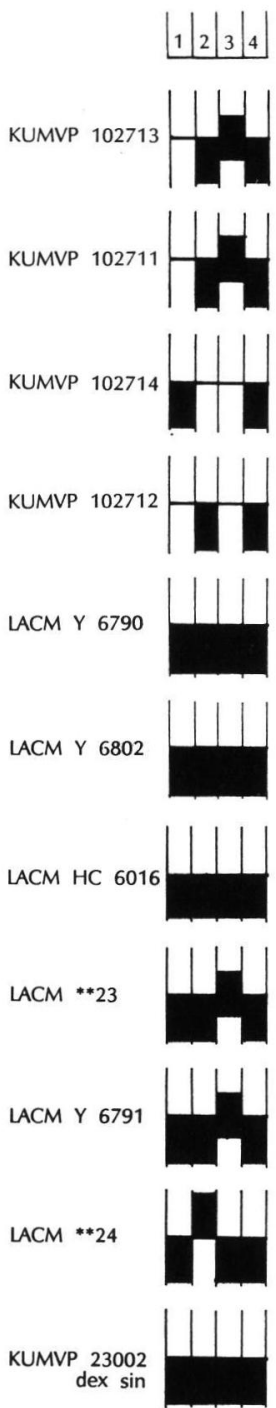

Abb. 17: Verteilungsmuster der Merkmalsausprägungen an der Tibia.

Feldnummern sind durch ein * gekennzeichnet oder durch Nummern mit ** vertreten (Zuordnung siehe 3.5); weitere Erklärung im Text.

Die am europäischen Bison priscus ausgearbeiteten Merkmale lassen sich mit gewissen Einschränkungen auch auf nordamerikanisches Bison-Material übertragen. Für eine Unterscheidung des nordamerikanischen Bison von eingeführten Hausrindern kommt jedoch erschwerend hinzu, daß Bos primigenius-typische Merkmale bei Bos primigenius f. taurus nicht konstant auftreten.

\section{Danksagung}

Herrn Prof. Dr. W. v. KoENIGswald, Institut für Paläontologie, Bonn, danke ich für zahlreiche Anregungen und Ratschläge sowie die kritische Durchsicht des Manuskriptes. Für die Möglichkeit, Material aus denen von ihnen betreuten Sammlungen zu bearbeiten, danke ich folgenden Herren: J. BoscheINEN, 
Löbbecke-Museum und Aquarium in Düsseldorf; A. CURRENT, British Museum (Natural History) in London; Prof. Dr. O. v. FrISCH, Staatliches Naturhistorisches Museum in Braunschweig; Dr. R. HEIL, Hessisches Landesmuseum in Darmstadt; Dr. G. T. JefFerson, George C. Page Museum (Los Angeles County Museum), Los Angeles; Dr. L. D. MARTIN, Museum of Natural History, University of Kansas, Lawrence. Herr BosCHEINEN stellte die Vorlage zu Abb. 12 zur Verfügung und gab mir wie Herr. R. MEINECKE, Brühl, einen wichtigen Literaturhinweis. Herr G. OlesCHINSKI, Bonn, reproduzierte die Vorlage zu Abb. 2.

\section{Schriftenverzeichnis}

Aaris-S $\phi$ Rensen, K. \& Brinch Petersen, E. (1986): The Prejlerup Aurochs - an Archaeozoological Discovery from Boreal Denmark. - Nordic Late Quaternary Biology and Ecology. Striae, 24: 111-117, 6 Abb.; Uppsala.

ADAM, K. D. (1954): Die mittelpleistozänen Faunen von Steinheim an der Murr (Württemberg). - Quaternaria, 1: 131-144, 3 Abb.; Rom.

ANDerson, E. (1984): Who's who in the Pleistocene: A mammalian bestiary. - in: MARTIN, P. S. \& KuEIN, R. S. (eds.): Quaternary extinctions. A prehistoric revolution: 40-89, $18 \mathrm{Abb} ., 1 \mathrm{Tab} . ;$ Tucson (University of Arizona Press).

BiBIKOVA, V. I. (1958) Some distinguishing features in the bones of the genera Bison and Bos (in russisch). - Bjull. Moskovsko Obšccestvo ispytatelej Prirody N.S. Otdel Biologiceskij, 63 (6): 23-25, 25 Abb.; Moskau.

Bojanus, L. H. (1828): De uro nostrate eiusque sceleto commentatio. - Nov. Act. phys.-med. Acad. Caes. Leopold. Carol., 13 (2): 413-478, 24 Taf.; Bonn.

Boscheinen, J. (1985): Geologie/Paläontologie. — Jahrber. 1984 Löbbecke Museum und Aquarium: 52-54; Düsseldorf.

COPE, G. R. \& ANGUS, R. B. (1975): An ecological study of a temperate interlude in the middle of the last glaciation, band on fossil Coleoptera from Isleworth, Middlesex. - J. Anim. Ecol., 44: 365-391, 7 Abb., 2 Taf.; Oxford.

CUvier, G. (1836): Recherches sur les ossemens fossiles. Atlas, 2; Paris.

Degerb $\phi$ L, M. \& Fredskild, B. (1970): The Urus (Bos primigenius Bojanus) and Neolithic domesticated cattle (Bos taurus domesticus Linné) in Denmark. - Biol. Skr. Dan. Vidensk. Selsk., 17/1: 1-234; Kopenhagen.

DrIESCH, A. VON DEN (1976): Das Vermessen von Tierknochen aus vor- und frühgeschichtlichen Siedlungen. - Inst. Paläoanatomie, Domestikationsforsch. Gesch. Tiermed. Univ. München, 114 S.; München.
FIEDLER, H. (1907): Über Säugetierreste aus Braunschweigischen Torfmooren nebst einem Beitrag zur Kenntnis der osteologischen Geschlechtscharaktere des Rindsschädels. - Z. Anthrop. Ethnol. Urgesch., 4/5: 1-60, 24 Fig., 11 Abb., 1 Taf.; Berlin.

FISCHER, K.-H. (1965): Bisonreste (Bison schoetensacki voigtstedtensis ssp. $\mathrm{n}$.) aus den altpleistozänen Tonen von Voigtstedt in Thüringen. - Paläont. Abh., Abt. A., 2, H. 2/3: 363-378, 5 Abb., Taf. 8-12; Berlin.

Flerov, K. K. (1969): Die Bison-Reste aus den Kiesen von Süßenborn bei Weimar. - Paläont. Abh., Abt. A., 3, H 3/4: 489-520, 17 Abb., Taf. 11-20; Berlin.

Gilbert, B. M. \& Martin, L. D. (1984): Late Pleistocene Fossils of Natural Trap Cave, Wyoming, and the climatic model of extinction. - in: MARTIN, P. S. \& KIEIN, R. G. (eds.): Quaternary extinctions. A prehistoric revolution: 138-147, 3 Abb.; Tucson (University of Arizona Press).

GÖRGES, J. (1912): Niederrheinische Museen. Das Löbbecke-Museum in Düsseldorf. - Der Niederrhein, 1, 9: 143-144; Kempen/Rh

HÜnermanN, K. A. (1968): Der Schädel eines Auerochsen (Bos primigenius BOJANUS 1828) von Ober-Illnau, Kt. Zürich. - Vierteljahresschrift Naturf. Ges. Zürich, 113: 337-346, 5 Abb., 2 Tab.; Zürich.

KAHLKE, H. D. (1982): Hippopotamus amphibius Des. MAREST, 1822 aus dem Pleistozän von Meiningen in Südthüringen (Bezirk Suhl). $-Z$. geol. Wiss., 10/7: 943-949, 2 Abb.; Berlin.

KoENIGSWALD, W. v. (1988): Paläoklimatische Aussage letztinterglazialer Säugetiere aus der nördlichen Oberrheinebene. - in: KoENigswald, W. v. (ed.): Zur Paläoklimatologie des letzten Interglazials im Nordteil der Oberrheinebene. - Paläoklimaforschung, 4: 205-314, 51 Abb.; Stuttgart.

— \& ToBien, H. (1987): Bemerkungen zur Altersstellung der pleistozänen Mosbach-Sande bei Wiesbaden. Geol. Jb. Hessen, 115: 227-237, 2 Abb., 1 Tab.; Wiesbaden

KuRTÉn, B. (1968): Pleistocene Mammals of Europe. 317 S., 111 Abb., 15 Tab.; London (Weidenfeld \& Nicolson).

LANGER, W. (1974): Der Auerochs vom Hülser Berge. Die Heimat, Z. niederrhein. Kulturpflege, 45: 127-128, 3 Abb.; Krefeld.

LehmanN, U. (1949): Der Ur im Diluvium Deutschlands und seine Verbreitung. - Neues Jb. Mineral., Geol. Paläont., Abt. B, Geol.-Paläont., 90: 163-266, 6 Taf.; Stuttgart.

LEMPPENAU, U. (1964): Geschlechts- und Gattungsunterschiede am Becken mitteleuropäischer Wiederkäuer. - Diss. München, 40 S., 6 Abb., 11 Tab., 22 Taf.; München. 
Martin, L. D. \& Gilbert, B. M. (1978a): Excavations at Natural Trap Cave. - Trans. Nebraska Acad. Sci., 6: 107-115, 5 Abb.; Lincoln, Nebraska.

- \&- (1978b): An American lion, Panthera atrox, from Natural Trap Cave, north central Wyoming. - Contrib. Geol., 16, 2: 95-101, 4 Abb.; Laramie, Wyoming.

Martin, L. D., Gilbert, B. M. \& Daniel, B. A. (1977): A cheetah-like cat in the North American Pleistocene. Science, 165: 981-982, 1 Abb.; Washington, D.C.

-, Whetstone, K. N., Chorn, J. D. \& Frailey, C. D. (1979): Survey of Fossil Vertebrates from East-Central Kansas. - Kansas River Bank Stabilization Study. Prepared for U.S. Army Corps of Engineers, Kansas City District. 28 S., 4 Abb., Lawrence (University of Kansas).

MARTIN, TH. (1987): Artunterschiede an den Langknochen großer Artiodactyla aus dem Jungpleistozän Mitteleuropas. - Cour. Forsch.-Inst. Senckenberg, 96: 1-124, $134 \mathrm{Abb}$; Frankfurt a. M.

McDonald, J. N. (1981): North American Bison. Their Classification and Evolution. 316 S., 103 Abb., 75 Tab.; Berkeley, Los Angeles, London (University of California Press).

MeINECKE, R. (1985): Auerochsen lebten einst im Erfttal. Revier und Werk, 35, 190: S. 15; Köln.

Miller, W. E. \& Brotherson, J. D. (1979): Size variation in foot elements of Bison from Rancho La Brea. Contr. Sci., 323: 1-19, 24 Abb., 2 Tab.; Los Angeles (Nat. Hist. Mus. Los Angeles County).

MoHr, E. (1952): Der Wisent. - Neue Brehm-Bücherei, 74: 75 S., 42 Abb.; Leipzig (Akad. Verlagsges.).

Nickel, R., SCHUmmer, A. \& Seiferle, E. (1984): Lehrbuch der Anatomie der Haustiere, 1, Bewegungsapparat. 542 S., 517 Abb.; 5. Aufl.; Berlin und Hamburg (Parey).

Olsen, S. (1960): Postcranial sceletal characters of Bison and Bos. - Pap. Peabody Mus. Archaeol. Ethnol., 35, (4): 1-15, 14 Abb.; Cambridge, Mass. (Harvard University).

Rogers, R. A. \& Martin, L. D. (1984): The 12 Mile Creek site: A reinvestigation. - American Antiquity, Reports, 49 (4): 757-764, 2 Abb.; Washington, D.C.

\section{Nachtrag:}

Während der Drucklegung wurde ein weitgehend vollständiges Auerochsenskelett aus Sassenberg, Kreis Warendorf im Münsterland bekannt gemacht (LANSER 1990), das hier der Vollständigkeit halber erwähnt werden soll, in die Untersuchungen aber nicht mehr einbezogen werden konnte. Das besonders starke Skelett stammt aus dem Aushub eines Teiches am Rand der Niederterrasse der Hessel (Nebenfluß der Ems) und hat nach einer Radiokarbon-Datierung meso-
RƯTIMEYER, L. (1862): Die Fauna der Pfahlbauten der Schweiz. - Neue Denkschr. allg. Schweiz. Ges. ges. Naturwiss., 19: 56-112, 6 Taf.; Zürich.

SCHERTZ, E. (1936): Zur Unterscheidung von Bison priscus (Boj.) und Bos primigenius Boj. an Metapodien und Astragalus, nebst Bemerkungen über einige diluviale Fundstellen. - Senckenbergiana, 18: 37-71, 17 Abb.; Frankfurt a. M.

SCHWEISS, D. (1988): Jungpleistozäne Sedimentation in der nördlichen Oberrheinebene. - in: KoENIGSWALD, W. v. (ed.): Zur Paläoklimatologie des letzten Interglazials im Nordteil der Oberrheinebene. - Paläoklimaforschung, 4: 19-78, 12 Abb.; Stuttgart.

SKINNER, M. F. \& KAISEN, O. C. (1947): The fossil Bison of Alaska and preliminary revision of the genus Bison. Bull. Amer. Mus. Nat. Hist. 89: 123-256, 5 Abb., Taf. 8-26, 25 Tab., 3 Kt.; New York.

StAmpfl, H. R. (1963): Wisent, Bison bonasus, Ur, Bos primigenius und Hausrind, Bos taurus. - In: BOESSNeCK, J., Jequier, J. P. \& Stampfli, H. R. (Hrsg.): Seeberg Burgäschisee-Süd. Die Tierreste, 3: 117-196, Fig. 6-42. - Acta Bernensia, 2: Bern.

Stock, CH. (1972): Rancho La Brea. A record of Pleistocene life in California. - Los Angeles County Museum Nat. Hist., Sci. Ser. 20, Paleontology, 11. 81 S., 33 Abb.; Los Angeles.

StUART, A. J. (1981): Pleistocene Vertebrates in the British Isles. - $121 \mathrm{~S}$, $163 \mathrm{Abb} ., 17 \mathrm{Tab}$; London und New York (Longman).

Sutcliffe, A. J. (1985): On the track of Ice Age mammals. 224 S, 139 Abb., 9 Tab.; London, British Museum (Natural History).

WIEGANK, F. (1983): Beitrag zur Chronostratigraphie und Entwicklung der Großsäugerfaunen im jüngeren Känozoikum von Europa auf geochronologischer und magnetostratigraphischer Grundlage. - Schriftenr. geol. Wiss., 19/20: 355-380, 2 Tab.; Berlin.

Wilson, M. V. (1974): The Caspar Site local fauna and its fossil bison. - in: Frison, G. C. (ed.): The Caspar Site: A hell gap bison kill on the High Plains: 125-171, 23 Abb., 15 Tab.; New York (Academic Press).

Zeuner, F. E. (1967): Geschichte der Haustiere. 448 S., 331 Abb.; München, Basel, Wien (BLV).

Manuskript eingegangen am 7. 11. 1989.

lithisches Alter; es befindet sich im Westfälischen Museum für Naturkunde in Münster.

LANSER, P. (1990): Der Fund eines Ur-Skelettes bei Sassenberg im Münsterland. - in: HeLlenKEMPER, $\mathrm{H}$. et al. (Hrsg.): Archäologie in Nordrhein-Westfalen. Geschichte im Herzen Europas. - Schriften zur Bodendenkmalpflege in Nordrhein-Westfalen, 1: 126-131, 6 Abb.; Mainz (Zabern). 